\title{
Confined axisymmetric laminar jets with large expansion ratios
}

\author{
By A. REVUELTA ${ }^{1}$, A. L. SÁNCHEZ ${ }^{1}$ AND A. LIÑ ÁN ${ }^{2}$ \\ ${ }^{1}$ Area de Mecánica de Fluidos, Departamento de Ingeniería Mecánica, \\ Universidad Carlos III de Madrid, 28911 Leganés, Spain \\ ${ }^{2}$ Departamento de Motopropulsión y Termofluidodinámica, E. T. S. I. Aeronáuticos, \\ Universidad Politécnica de Madrid, 28040 Madrid, Spain
}

(Received 26 September 2000 and in revised form 17 October 2001)

This paper investigates the steady round laminar jet discharging into a coaxial duct when the jet Reynolds number, $R e_{j}$, is large and the ratio of the jet radius to the duct radius, $\varepsilon$, is small. The analysis considers the distinguished double limit in which the Reynolds number $R e_{a}=R e_{j} \varepsilon$ for the final downstream flow is of order unity, when four different regions can be identified in the flow field. Near the entrance, the outer confinement exerts a negligible influence on the incoming jet, which develops as a slender unconfined jet with constant momentum flux. The jet entrains outer fluid, inducing a slow backflow motion of the surrounding fluid near the backstep. Further downstream, the jet grows to fill the duct, exchanging momentum with the surrounding recirculating flow in a slender region where the Reynolds number is still of the order of $R e_{j}$. The streamsurface bounding the toroidal vortex eventually intersects the outer wall, in a non-slender transition zone to the final downstream region of parallel streamlines. In the region of jet development, and also in the main region of recirculating flow, the boundary-layer approximation can be used to describe the flow, while the full Navier-Stokes equations are needed to describe the outer region surrounding the jet and the final transition region, with $R e_{a}=R e_{j} \varepsilon$ entering as the relevant parameter to characterize the resulting non-slender flows.

\section{Introduction}

Confined jet flows are of both fundamental and practical importance. They are present in numerous applications including ejector systems and gas-turbine combustors. A prototypical example of such flows arises in axisymmetric ducted flows with sudden expansions; the flow separates as it encounters the expansion, comprising a jet stream surrounded by recirculating flow. In this geometrically simple configuration, which is sketched in figure 1, the flow depends mainly on two parameters: the Reynolds number of the incoming jet, $R e_{j}$, and the expansion ratio, $1 / \varepsilon$, with $\varepsilon$ representing the ratio of the inner to the outer radii; the laminar jet remains stable for values of the Reynolds number below a certain critical value. The purpose of the present paper is to describe the resulting steady axisymmetric solutions in the double limit of large Reynolds numbers $\left(R e_{j} \gg 1\right)$ and large expansion ratios $(\varepsilon \ll 1)$. The analysis considers in particular the distinguished limit $\operatorname{Re}_{j} \varepsilon \sim 1$, for which the Reynolds number $R e_{a}=R e_{j} \varepsilon$ of the asymptotic flow emerging downstream from the recirculating region is of order unity. In the computations, both uniform and Poiseuille profiles will be considered for the inlet velocity profile. Also, the boundary 


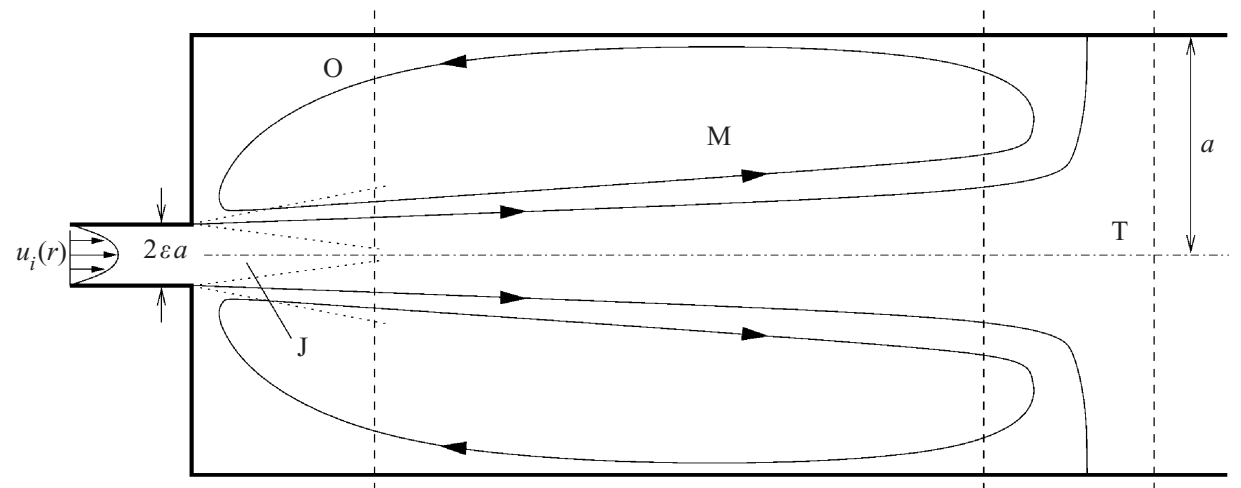

Figure 1. A schematic of the confined jet flow.

conditions on the outer wall will include both non-slip and slip flow, the latter being an appropriate symmetry condition to represent approximately the collective effect of the other jets in array configurations of multiple jets. Before focusing on the problem of interest, we review below some relevant results concerning the solution for round laminar jets. Although stability is not the subject of the present paper, a brief account of our current understanding of the stability of confined jets is also provided.

The flow field in unconfined jets $(\varepsilon=0)$ emerging normal to a wall depends only on the value of $R e_{j}$. A uniformly valid exact solution of the resulting flow is not available. If $R e_{j} \sim 1$, one needs to integrate the full Navier-Stokes equations to describe the flow field near the mouth of the pipe, i.e. at distances from the orifice of the order of its radius. The flow field has been described in jets with $R e_{j} \gg 1$. In this case, there exists a slender jet region aligned with the pipe where the velocity is of the order of the inlet velocity, and where the momentum flux is constant in the first approximation, because the velocity outside is much smaller. This jet develops downstream as it entrains outer fluid. The boundary-layer approximation is applicable to describe the jet flow, whereas the full Navier-Stokes equations are necessary to study the motion outside. At distances from the entrance much larger than $R e_{j}$ times the pipe radius, the flows in the jet and in the outer region become self-similar. In the jet region, the solution approaches that postulated by Schlichting (1933), corresponding to a point source of momentum. The jet is seen to entrain outer fluid with a radial volume flux per unit length equal to $8 \pi v$, with $v$ denoting here the kinematic viscosity of the fluid. This constant entrainment rate, independent of the jet Reynolds number, is associated with a Reynolds-number-independent self-similar solution in the outer Navier-Stokes region, a result due to Schneider (1981), who later used a multiple-scale technique to account for the slow momentum decay that occurs in the jet in the presence of the outer flow (Schneider 1985). At large distances from the inlet the jet has lost a significant fraction of its initial momentum, but is still slender, and continues to entrain outer fluid with the same constant rate $8 \pi v$, at distances from the inlet smaller than $R e_{j} \exp \left(R e_{j}^{2} / 15.28\right)$ times the pipe radius. At these distances, the jet merges with the outer flow, giving rise to a large recirculating toroidal eddy whose description necessitates numerical integration of the full NavierStokes equations. The associated parameter-free problem has not been investigated, although an approximate description, obtained by extending the near-field asymptotic expansion outside its range of applicability, is given in Schneider (1985), with results in agreement with experimental observations (Zauner 1985). 
The flow-field structure in confined jets is drastically different from that described by Schneider (1985). The problem has been investigated by several authors, although the limit of large expansion ratios studied here has not been thoroughly considered before. For values of the jet Reynolds number sufficiently larger than unity, the resulting steady symmetric jet becomes slender, with a structure that, in most of the flow field, can be investigated with the boundary-layer approximation. Use of appropriate scales then enables the problem to be written in a dimensionless form independent of the Reynolds number. The boundary-layer description of confined jet flows was attempted by Kumar \& Yajnik (1980), who employed an expansion in eigenfunctions of the Poiseuille flow to approximately solve the planar sudden-expansion problem. More extensive boundary-layer solutions were computed by Acrivos \& Schrader (1982) and Milos \& Acrivos (1986) for both planar and axisymmetric configurations with different expansion ratios. They found that, while solutions can always be obtained for parabolic velocity profiles, the solution with a uniform inlet profile may only exist for expansion ratios above a critical value, when the pressure gradient becomes singular near the reattachment point. This intriguing characteristic of confined jets was further investigated by Milos \& Acrivos (1987), who performed integrations of the Navier-Stokes equations for values of the expansion ratio approaching the critical value. Although their analysis was restricted for simplicity to a planar configuration with slip flow on the outer boundary, as corresponds for instance to an infinite cascade of equally spaced jets, their computations clearly demonstrated that the failure of the boundary-layer approximation for small expansion ratios is associated with the existence of non-slender recirculating regions in these large-Reynolds-number flows.

Acrivos and coworkers (Acrivos \& Schrader 1982; Milos \& Acrivos 1986) also uncovered a subtle point concerning the use of the boundary-layer approximation for the description of the sudden-expansion recirculating flows considered in their work. The effect of viscosity was not able to arrest the recirculating fluid that moves upstream, giving rise to the existence of a non-slender turnaround inviscid region in the vicinity of the backstep. When solving the sudden-expansion flow with the boundary-layer approximation, non-zero velocities must be considered in general at the backstep wall to ensure matching with the backstep region. The presence of this so-called collision region is a complicating characteristic of the boundary-layer description that emerges in other slender large-Reynolds-number flows when reverse flow is present (Klemp \& Acrivos 1976; Brady \& Acrivos 1982).

In the double limit $\varepsilon \ll 1$ and $R e_{j} \gg 1$ with $R e_{a}=R e_{j} \varepsilon \sim 1$, which we analyse here, four different regions can be identified in the flow field, as indicated in the sketch of figure 1 . Near the entrance, there is a slender region $(\mathrm{J})$ of jet development with a length $l_{j}$ of the order of $R e_{j}$ times the jet radius $\varepsilon a$. The jet entrainment induces a motion of the surrounding fluid located near the backstep, as in the case of unconfined jets studied by Schneider (1981). Farther downstream, there exists a main recirculating region ( $\mathrm{M}$ in the schematic), where the motion is determined in the first approximation by the momentum flux of the incoming jet. The velocities in this region are $\varepsilon$ times the inlet jet velocity, while the corresponding Reynolds number remains of the order of $R e_{j}$. In this slender region $\mathrm{M}$, with a characteristic length $l_{m}$ equal to $R e_{j}$ times the confining pipe radius $a$, there is a recirculating eddy that can be described at leading order with the boundary-layer approximation. Downstream from this eddy the flow approaches an asymptotic parallel flow, determined by the mass flux of the incoming jet, with characteristic velocities that are $\varepsilon^{2}$ times the inlet jet velocity and with a characteristic Reynolds number given by $R e_{a}=\varepsilon R e_{j}$. Clearly, since the asymptotic flow velocity is a factor $\varepsilon$ smaller than that found in 
$\mathrm{M}$, the description of the recirculating eddy in the limit $\varepsilon \rightarrow 0$ must show at leading order a stagnant solution downstream from the eddy end, with non-zero velocities of relative order $\varepsilon$ appearing downstream only at the following order in the asymptotic development.

The streamlines of the slender recirculating eddy of length $l_{m}=R e_{j} a$ are aligned with the axis, except in non-slender boundary regions of characteristic length $a$ located at both ends $(\mathrm{O}$ and $\mathrm{T}$ in the schematic). In the outer region $\mathrm{O}$ surrounding the incoming jet, the streamlines deflect towards the axis forced by the jet entrainment. If $R e_{a}$ is of order unity, which corresponds to the distinguished limit $\varepsilon R e_{j} \sim 1$ considered here, then the length of jet development $l_{j}=\operatorname{Re}_{a} a$ and the length of the non-slender region $\mathrm{O}$ are comparable; this is the case represented in the schematic of figure 1 . The leading-order solution in this outer region $\mathrm{O}$, and also in the final transition region $\mathrm{T}$, will be described for various values of $R e_{a} \sim 1$, with the extreme cases $R e_{a} \ll 1$ and $R e_{a} \gg 1$ also being considered. The downstream asymptotic forms of the solutions for the regions $\mathrm{O}$ and $\mathrm{J}$ provide in particular the velocity profile for the integration of the boundary-layer equations in the main recirculating region; no turnaround, or collision, region is found at the backstep in the analysis of sudden expansions with $\varepsilon \ll 1$.

The stability analysis of confined jets should provide, in particular, the maximum value of $R e_{j}$ for which the steady laminar solution remains valid. Much of the stability research on confined jets has been devoted to the case of plane sudden expansions. For instance, Durst, Melling \& Whitelaw (1974) and Cherdron, Durst \& Whitelaw (1978) studied this problem experimentally, and found that symmetric solutions can only exist for values of the Reynolds number below a certain critical value. For larger values of the Reynolds number, steady asymmetric solutions appear, a result that was confirmed in the numerical works of Durst, Pereira \& Tropea (1993) and Allerborn et al. (1997). Recent contributions regarding the symmetry-breaking bifurcation include that of Rusak \& Hawa (1999), who carried out a weakly nonlinear analysis of the bifurcation, and that of Hawa \& Rusak (2000), who studied the effect of a slight asymmetry of the channel geometry on the flow behaviour.

The symmetry-breaking bifurcation was also encountered in the numerical and experimental work of Fearn, Mullin \& Cliffe (1990), who investigated a plane sudden expansion with expansion ratio $1: 3$. They obtained a critical Reynolds number (based on the jet width) equal to 82 , a result later verified by the linear stability analysis of Shapira, Degani \& Weihs (1990). For even larger values of the Reynolds number, the experimental evidence indicates that the flow becomes time-dependent, a behaviour that Fearn et al. (1990) and Durst et al. (1993) found to be associated with three-dimensional effects. The dependence of the critical Reynolds number of the symmetry-breaking bifurcation on the expansion ratio was studied in the more recent numerical works of Battaglia et al. (1997) and Drikakis (1997). It was found that reducing the expansion ratio tends to improve the stability of the symmetric solution; the critical Reynolds number decreases with increasing expansion ratios. The interplay of viscous dissipation and convection of perturbations for increasing jet Reynolds number is discussed in detail by Hawa \& Rusak (2001), who combined a bifurcation analysis and a linear stability study of the sudden expansion with careful direct numerical simulations of the problem.

The stability of axisymmetric free jets was addressed in the early experimental work of Reynolds (1962). Steady solutions were found for Reynolds numbers (based on the jet diameter) below about 300 . Confinement does not greatly affect this critical value; in a free round jet the local Reynolds number remains constant with axial 
distance (Schlichting 1933), causing jet stability in sudden expansions to remain roughly independent of the expansion ratio. For instance, in the experimental and numerical work of Macagno \& Hung (1967), for an expansion ratio 1:2 steady solutions were found for jet Reynolds numbers at least as large as 200.

Although the stability analysis of unconfined round jets was undertaken early by Batchelor \& Gill (1962) and by Mollendorf \& Gebhart (1973), much remains to be learnt about the stability of confined round jets. The stability behaviour can be expected to be different from that described above for plane jets. The experimental results for turbulent jets in sudden expansions of Nathan, Hill \& Luxton (1998) suggest that, while the steady axisymmetric solution in planar jets undergoes a symmetrybreaking bifurcation to another steady solution, round jets may bifurcate to unsteady asymmetric solutions, in which the jet precesses about the axis in a swirl-like motion. Although this type of behaviour is anticipated by Battaglia et al. (1998), careful evaluations of the critical Reynolds numbers associated to this bifurcating mode are still not available. Another aspect of the problem in need of further research concerns the relationship between the stability characteristics of confined and unconfined jets.

\section{Characteristic scales and problem formulation}

We consider here the confined laminar jet formed when an incompressible fluid of density $\rho$ and kinematic viscosity $v$ flows through a pipe of radius $\varepsilon a$ into a much larger coaxial pipe of radius $a$, a configuration sketched in figure 1 . The momentum and mass fluxes of the incoming jet are, respectively, $\mathrm{J}=\int_{0}^{\varepsilon a} 2 \pi \rho r u_{i}^{2} \mathrm{~d} r$ and $\mathrm{G}=\int_{0}^{\varepsilon a} 2 \pi \rho r u_{i} \mathrm{~d} r$, where $r$ is the radial distance to the axis and $u_{i}$ is the incoming velocity distribution. In our analysis, we shall assume that the jet Reynolds number $R e_{j}=(\mathrm{J} / \rho)^{1 / 2} / v$ is much larger than unity. Furthermore, attention is restricted to cases with large expansion ratios $(\varepsilon \ll 1)$, for which different distinguished regions can be identified in the flow field sketched in figure 1. In the following discussion of characteristic scales, the variable $x$ denotes the axial distance measured from the entrance, $u$ and $v$ are the axial and radial velocity components and $p$ denotes the pressure, all variables being expressed in dimensional form.

Close to the entrance, the outer confinement exerts a negligible influence on the jet, which thereby behaves as an unconfined free jet, with a momentum flux that remains constant in the first approximation. In this jet development region, denoted by $\mathrm{J}$ in figure 1 , the velocity is of order $u_{j}=(\mathrm{J} / \rho)^{1 / 2} /(\varepsilon a)$ and the jet radius is of order $\varepsilon a$. An order-of-magnitude balance between the viscous terms, $v u_{j} /(\varepsilon a)^{2}$, and the convective terms, $u_{j}^{2} / l_{j}$, in the jet momentum equation yields $l_{j}=R e_{j} \varepsilon a$ for the characteristic length of this initial region of jet development. Clearly, the condition that the jet Reynolds number $R e_{j}$ be large ensures that the resulting jet is slender, and can consequently be described in the boundary-layer approximation. Further downstream, the solution in the jet approaches the Schlichting solution (Schlichting 1933), with characteristic values for the local jet radius $\varepsilon a x / l_{j}$ and axial velocity $u_{j} l_{j} / x$ that follow from the condition of constant momentum flux and from the balance between viscous forces and convective terms in the momentum equation. The jet volume flux increases with distance due to the entrainment of outer fluid, with a radial volume flux per unit length $2 \pi r v$ of order $v$ that decreases towards the constant value $8 \pi v$ in the self-similar Schlichting region.

The jet therefore acts as a volumetric line sink that induces the motion of the surrounding fluid located near the backstep wall between the jet and $r=a(\mathrm{O}$ in the figure), with axial and radial velocities of order $u_{o} \sim \varepsilon^{2} u_{j}$ and $v_{o} \sim v / a$ at distances 
$x \sim l_{j}$. In the distinguished limit $R e_{a}=R e_{j} \varepsilon \sim 1$, the outer region $\mathrm{O}$ is non-slender, i.e. $l_{j} \sim a$ and $u_{o} \sim v_{o}$. The description of the associated velocity field requires integration of the full Navier-Stokes equations, with $R e_{a}$ entering as a parameter in the formulation. On the other hand, if $R e_{a} \gg 1$, then the streamlines remain almost parallel to the axis in $\mathrm{O}$ for $x \sim l_{j} \sim R e_{a} a$, where the flow can be described with the boundarylayer approximation, except in the small non-slender region of streamline deflection corresponding to $x \sim a$. In the opposite limit $R e_{a} \ll 1$, the length of jet development satisfies $l_{j} \ll a$, so that to study the fluid motion in $\mathrm{O}$ at distances $x \sim a$ one can use the constant jet entrainment at the axis $8 \pi v$ corresponding to Schlichting solution.

Farther downstream from the entrance there exists a much larger region $(\mathrm{M}$ in the schematic) characterized by a momentum exchange between the jet and the outer recirculating fluid. Correspondingly, the characteristic axial velocity in this main region, $u_{m}=(\mathrm{J} / \rho)^{1 / 2} / a=\varepsilon u_{j}$, follows from the condition $\mathrm{J} \sim \rho u_{m}^{2} a^{2}$. Note that this condition of momentum exchange also implies that the characteristic Reynolds number in $\mathrm{M}$ is of the order of the jet Reynolds number, i.e. $u_{m} a / v=R e_{j}$. On the other hand, the balance between the viscous term $v u_{m} / a^{2}$ and the convective term $u_{m}^{2} / l_{m}$ in the momentum balance equation yields $l_{m}=R e_{j} a$ for the characteristic length of the recirculating region, whereas the continuity balance $u_{m} / l_{m} \sim v_{m} / a$ leads to a characteristic radial velocity in this region $v_{m}=v / a \sim u_{m} / R e_{j}$. These characteristic values $u_{m}, v_{m}$ and $l_{m}$, together with the radius $a$ and the characteristic pressure variation $\rho u_{m}^{2}$, are used below as scales in writing the conservation equations in dimensionless form. As shown below, in the limiting case $R e_{j} \gg 1$ considered here, these equations reduce, with errors of order $R e_{j}^{-2}$, to the well-known boundary-layer equations. Note that, in the case of unconfined jets investigated by Schneider (1985), one needs a jet length of order $\varepsilon a R e_{j} \exp \left(R e_{j}^{2} / 15.28\right) \gg l_{m}$ for the momentum to decay to negligible values.

At the rear end of the recirculating region the dividing streamsurface opens up and eventually intersects the outer wall. The flow downstream rapidly approaches either Poiseuille flow (if a non-slip condition is employed at $r=a$ ) or a uniform flow (if slip flow is considered). Mass conservation requires that the characteristic axial velocity in this asymptotic downstream region be of order $\mathrm{G} /\left(\rho a^{2}\right) \sim \varepsilon^{2} u_{j} \sim \varepsilon u_{m}$, and that the corresponding asymptotic Reynolds number be $R e_{a}=R e_{j} \varepsilon$. Transition between the recirculating flow and the final downstream parallel flow takes place in a short region (denoted by $\mathrm{T}$ in figure 1 ) of characteristic length $\operatorname{Re}_{a} a$ where the velocity is already of order $\varepsilon^{2} u_{j}$, and where the local Reynolds number is $R e_{a}$. As in region $\mathrm{O}$, the description of the flow field in $\mathrm{T}$ requires integration of the Navier-Stokes equations, with $R e_{a}$ entering as a parameter.

The scaling analysis can be extended to estimate the pressure variations taking place in the different regions. Thus, in the boundary regions $\mathrm{O}$ and $\mathrm{T}$ the pressure variations are of order $\rho \varepsilon^{4} u_{j}^{2}$, much smaller than the axial pressure differences found along the main region $\mathrm{M}$, of order $\rho \varepsilon^{2} u_{j}^{2}$. On the other hand, since the initial momentum per unit volume in the jet is of order $\rho u_{j}^{2}$, the pressure differences in $\mathrm{O}$ are much too small to affect the development of the jet at leading order. Therefore, in region $\mathrm{J}$ the velocity field can be computed using the boundary-layer approximation with the pressure gradient neglected when writing the axial momentum equation. The radial pressure differences across the jet, of order $\rho u_{j}^{2} / R e_{j}^{2}$, can be determined in region J by integrating the radial component of the momentum equation once the velocity field has been computed with the boundary-layer approximation.

The existence of four distinguished regions is a direct consequence of the large expansion ratio considered here; the boundary regions $\mathrm{J}, \mathrm{O}$ and $\mathrm{T}$ merge with the main recirculating region $\mathrm{M}$ in configurations with $\varepsilon$ of order unity. In the jet region, 
and also in the main recirculating region, the condition $R e_{j} \gg 1$ assumed here suffices to guarantee that the flow field is slender, and that it is amenable to a boundary-layer description. On the other hand, the solution in the non-slender regions $\mathrm{O}$ and $\mathrm{T}$ requires integration of the full Navier-Stokes equations, with $R e_{a}=\varepsilon R e_{j}$ entering as a parameter in the formulation. The following four sections deal with the leadingorder solution corresponding to regions $\mathrm{J}, \mathrm{O}, \mathrm{M}$ and $\mathrm{T}$, respectively. The analysis of the main region will be extended to account for the first-order corrections, and the results will be compared with integrations of the full Navier-Stokes equations, which are written in non-dimensional form below.

Using as scales for the different flow variables those corresponding to the main recirculating region, the governing equations take the dimensionless form

$$
\begin{gathered}
\frac{\partial u}{\partial x}+\frac{1}{r} \frac{\partial(r v)}{\partial r}=0 \\
u \frac{\partial u}{\partial x}+v \frac{\partial u}{\partial r}=-\frac{\partial p}{\partial x}+\frac{1}{r} \frac{\partial}{\partial r}\left(r \frac{\partial u}{\partial r}\right)+\frac{1}{R e_{j}^{2}} \frac{\partial^{2} u}{\partial x^{2}}, \\
\frac{1}{R e_{j}^{2}}\left(u \frac{\partial v}{\partial x}+v \frac{\partial v}{\partial r}\right)=-\frac{\partial p}{\partial r}+\frac{1}{R e_{j}^{2}} \frac{\partial}{\partial r}\left(\frac{1}{r} \frac{\partial(r v)}{\partial r}\right)+\frac{1}{R e_{j}^{4}} \frac{\partial^{2} v}{\partial x^{2}},
\end{gathered}
$$

where $r=r / a, x=x /\left(\operatorname{Re}_{j} a\right), u=u / u_{m}, v=v / v_{m}$, and $p=p /\left(\rho u_{m}^{2}\right)$. The boundary conditions at $x=0$ are

$$
0 \leqslant r \leqslant \varepsilon: \quad u=\varepsilon^{-1} U_{i}, \quad v=0
$$

and

$$
\varepsilon<r \leqslant 1: \quad u=v=0 .
$$

According to the scaling employed here, the jet velocity at the entrance, $u=\varepsilon^{-1} U_{i}$, is of order $1 / \varepsilon$. The function $u_{i} / u_{j}=U_{i}(r)$, of order unity, gives the normalized shape of the inlet velocity profile, with limiting cases of practical interest being the uniform velocity distribution $U_{i}=\pi^{-1 / 2}$ and the fully developed profile $U_{i}=(3 / \pi)^{1 / 2}\left[1-(r / \varepsilon)^{2}\right]$. Note that the boundary condition (2.4) is only appropriate for the cases $R e_{j} \gg 1$ considered here. In general, perturbations to the flow in the pipe upstream from the exit orifice should be accounted for, but these perturbations become negligible when the Reynolds number in the pipe is sufficiently large.

For $x>0$ the solution must satisfy the symmmetry condition at the axis

$$
r=0: \quad \partial u / \partial r=v=0 .
$$

Additional boundary conditions are, for $x>0$,

$$
r=1: \quad u=v=0
$$

if non-slip flow is assumed at the outer wall and

$$
r=1: \quad \partial u / \partial r=v=0
$$

for slip flow. As an additional boundary condition, far downstream the flow must approach either the Poiseuille profile for non-slip flow or a uniform flow with slip:

$$
x \gg 1: \begin{cases}u=\left(2 g_{o} / \pi\right)\left(1-r^{2}\right) \varepsilon, v=0 & \text { (for non-slip flow) } \\ u=\left(g_{o} / \pi\right) \varepsilon, v=0 & \text { (for slip flow) }\end{cases}
$$

with $g_{o}$ being a constant of order unity that depends on the shape function $U_{i}$. 
Relevant values corresponding to uniform flow and to Poiseuille flow are, respectively, $g_{o}=\pi^{1 / 2}$ and $g_{o}=(3 \pi)^{1 / 2} / 2$. In terms of the variables defined here, the momentum flux of the inlet velocity profile (2.4) is seen to satisfy $\int_{0}^{\varepsilon} 2 \pi r \varepsilon^{-2} U_{i}^{2} \mathrm{~d} r=1$, whereas its associated mass flow rate, $\mathrm{G}$, gives

$$
\frac{\mathrm{G}}{\rho^{1 / 2} \mathrm{~J}^{1 / 2} a}=\int_{0}^{\varepsilon} 2 \pi r \varepsilon^{-1} U_{i} \mathrm{~d} r=g_{o} \varepsilon
$$

Aside from the shape function $U_{i}$, the solution depends only on the expansion ratio $\varepsilon$ and on the jet Reynolds number $R e_{j}$.

It is worth pointing out that the present analysis is only applicable to configurations in which the outer duct is longer than the resulting recirculating region, yielding flow fields like that sketched in figure 1 . Since in this case the pressure at the exit section is equal to the ambient pressure, the duct length enters in the problem by determining the pressure level, but it is otherwise irrelevant. The description given below is no longer valid when the computed recirculating region is shorter than the duct. Then, fluid enters the duct from outside, giving a complicated non-slender flow pattern in the duct, whose description is outside the scope of the present paper.

\section{The jet region}

The scalings previously identified for the region of jet development, namely, $r \sim \varepsilon a$, $x \sim l_{j}, u \sim u_{j}$ and $v \sim v /(\varepsilon a)$, yield $X=x / \varepsilon, R=r / \varepsilon, U_{j}=\varepsilon u$, and $V_{j}=\varepsilon v$ as appropriate rescaled variables to analyse the jet region J. Introduction of these variables allows (2.1) and (2.2) to be written, with small relative errors of order $R e_{j}^{-2}$, in the form

$$
\begin{gathered}
\frac{\partial U_{j}}{\partial X}+\frac{1}{R} \frac{\partial\left(R V_{j}\right)}{\partial R}=0, \\
U_{j} \frac{\partial U_{j}}{\partial X}+V_{j} \frac{\partial U_{j}}{\partial R}=\frac{1}{R} \frac{\partial}{\partial R}\left(R \frac{\partial U_{j}}{\partial R}\right),
\end{gathered}
$$

while the radial momentum balance, which is written below in (3.11) in terms of the jet variables, determines the small pressure changes that occur across the jet. Appropriate boundary conditions for this boundary-layer problem are

$$
\begin{gathered}
X=0 \quad\left\{\begin{aligned}
0 \leqslant R \leqslant 1: & U_{j}=U_{i}(R) \\
R>1: & U_{j}=0,
\end{aligned}\right. \\
X>0 \quad \begin{cases}R=0: & \partial U_{j} / \partial R=V_{j}=0 \\
R \rightarrow \infty: & U_{j}=0 .\end{cases}
\end{gathered}
$$

As previously discussed, the pressure differences that appear in the surrounding region $\mathrm{O}$ are too small to affect the jet motion in the first approximation, so that the axial pressure gradient is absent in (3.2).

The numerical integration of this problem for a given initial velocity profile $U_{i}(R)$ gives the evolution of the jet, which spreads downstream as it entrains outer fluid. The integration determines in particular the radial entrainment velocity by the jet, $\left(R V_{j}\right)_{R=\infty}=-\Phi(X)$, so that $2 \pi \Phi(X)$ corresponds to the radial volume flux entrained by the jet per unit length. Correspondingly, the jet volume flux $\int_{0}^{\infty} 2 \pi R U_{j} \mathrm{~d} R$ continuosly increases, from its initial value $\int_{0}^{1} 2 \pi R U_{i} \mathrm{~d} R=g_{o}$, according to the entrainment law

$$
\frac{\mathrm{d}}{\mathrm{d} X}\left(\int_{0}^{\infty} 2 \pi R U_{j} \mathrm{~d} R\right)=2 \pi \Phi(X)
$$


obtained by radial integration of (3.1). Similarly, from the above system of equations it is easy to show that the momentum flux

$$
\int_{0}^{\infty} 2 \pi R U_{j}^{2} \mathrm{~d} R=1
$$

remains constant.

The rate of entrainment $\Phi(X)$ corresponding to an initially uniform velocity profile $U_{i}=\pi^{-1 / 2}$ and that of a fully developed parabolic profile $U_{i}=(3 / \pi)^{1 / 2}\left[1-R^{2}\right]$ are shown in figure 2 . As expected, both curves asymptotically approach the value $\Phi(X)=4$ as the jet evolves to the self-similar Schlichting solution for increasing values of $X$, a transition that occurs at moderately small values of $X$, of order 0.1 . On the other hand, for $X \ll 1$ the entrainment rate is determined by the locally planar mixing layer that forms at $R=1$ between the jet and the outer stagnant fluid. In general, the initial entrainment rate depends on the wall value of the velocity gradient $A=-\mathrm{d} U_{i} / \mathrm{d} R$ of the jet. This gradient is $A=2(3 / \pi)^{1 / 2}$ for the parabolic profile and becomes larger for decreasing values of the boundary-layer thickness. The initial, Goldstein region of the mixing layer can be described by introducing a similarity variable $y=(R-1) /(X / A)^{1 / 3}$ together with a normalized stream function $G(y)$ defined such that $U_{j}=A^{2 / 3} X^{1 / 3} G_{y}$ and $V_{j}=A^{1 / 3} X^{-1 / 3}\left(y G_{y} / 3-2 G / 3\right)$. The problem reduces to that of integrating $G_{y y y}+2 G G_{y y} / 3-G_{y}^{2} / 3=0$ subject to the boundary conditions $G_{y}(y \rightarrow \infty)=0$ and $G+y^{2} / 2 \rightarrow 0$ as $y \rightarrow-\infty$ (Goldstein 1930). The boundary condition as $y \rightarrow-\infty$ comes from imposing that there are no changes in jet velocity in the first approximation, so that the mixing layer entrains fluid only from the stagnant side. The entrainment rate $\Phi=(2 / 3) G_{\infty} A^{1 / 3} X^{-1 / 3}$ is dictated by the value $G_{\infty} \simeq 1.258$ of the streamfunction at $y=\infty$; it grows with the cube root of the velocity gradient at the base of the boundary layer of the incoming jet. The analysis must however be modified when a uniform velocity profile $U_{i}=\pi^{-1 / 2}$ is considered. The appropriate similarity coordinate in that case is $y=(R-1) /\left(X / U_{i}\right)^{1 / 2}$ and the normalized streamfunction $G(y)$ must be defined to give $U_{j}=U_{i} G_{y}$ and $V_{j}=$ $U_{i}^{1 / 2} X^{-1 / 2}\left(y G_{y} / 2-G / 2\right)$. The resulting equation, $G_{y y y}+G G_{y y} / 2=0$, must be integrated with boundary conditions $G_{y}(y \rightarrow \infty)=0$ and $G-y \rightarrow 0$ as $y \rightarrow-\infty$, a problem solved by Chapman (1949) and Lessen (1950). The corresponding entrainment rate becomes $\Phi=(1 / 2) G_{\infty} U_{i}^{1 / 2} X^{-1 / 2}$, where $G_{\infty} \simeq 1.238$ is computed from the above problem. In summary, the entrainment rate for $X \ll 1$ varies in general according to $\Phi=C X^{-\alpha}$, where $C \simeq 1.049$ and $\alpha=1 / 3$ for a Poiseuille jet profile and $C \simeq 0.465$ and $\alpha=1 / 2$ for a uniform profile. These asymptotic forms of the entrainment rate for $X \ll 1$ are included for completeness in the plots shown in figure 2 .

For $X \gg 1$ the solution to (3.1)-(3.4) approaches a self-similar solution in which the jet acts in the first approximation as a point source of momentum (Schlichting 1933). The condition of constant momentum flux, together with simple order-ofmagnitude estimates in (3.1) and (3.2), indicate that the radius of the jet increases linearly with distance, while the velocity components decrease according to $U_{j} \propto X^{-1}$ and $V_{j} \propto X^{-1}$. These scalings motivate the introduction of a similarity coordinate $\bar{\eta}=R / X$, together with expansions in decreasing powers of $X$ of the form $U_{j}=$ $X^{-1} U_{j}^{1}(\bar{\eta})+X^{-2} U_{j}^{2}(\bar{\eta})+\cdots$ and $V_{j}=X^{-1} V_{j}^{1}(\bar{\eta})+X^{-2} V_{j}^{2}(\bar{\eta})+\cdots$. Introducing these new variables into (3.1) and (3.2) gives a sequence of ordinary differential equations to be integrated with boundary conditions $\mathrm{d} U_{j}^{k} / \mathrm{d} \bar{\eta}=V_{j}^{k}=0$ at $\bar{\eta}=0$ and $U_{j}^{k}=0$ as $\bar{\eta} \rightarrow \infty$. As explained by Revuelta, Sánchez \& Liñán (2001), the solution for the first two terms in the expansion can be expressed jointly by introducing an apparent 

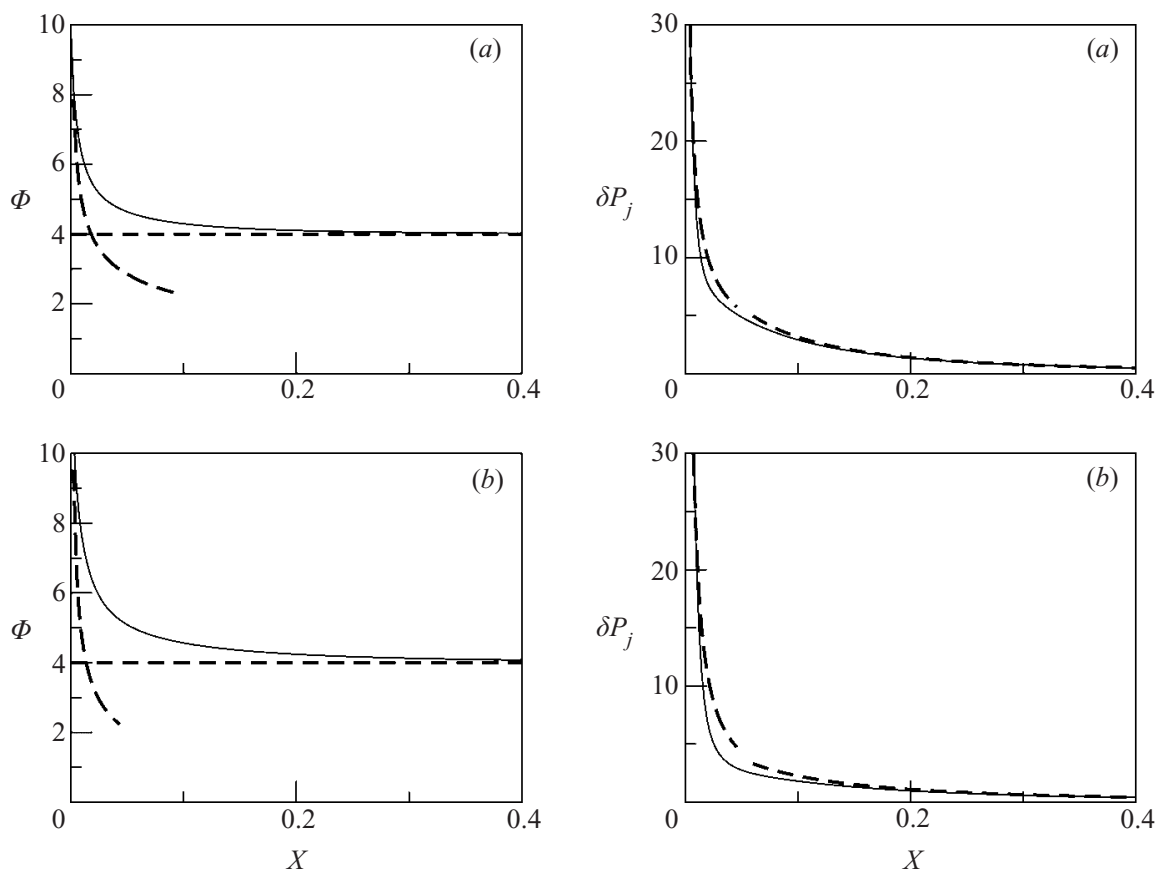

Figure 2. The function $\Phi(X)$ (left-hand plots) and the pressure jump $\delta P_{j}(X)$ (right-hand plots) for a fully developed parabolic profile $(a)$ and for a initially uniform velocity profile $(b)$; dashed lines represent the asymptotic behaviors for $X \ll 1$ and $X \gg 1$.

origin $X_{O}$ in the axial coordinate, a development that yields

$$
U_{j}=\frac{1}{X+X_{O}} \frac{512 \pi / 3}{\left(64 \pi / 3+\eta^{2}\right)^{2}}, \quad V_{j}=\frac{1}{X+X_{O}} \frac{4 \eta\left(64 \pi / 3-\eta^{2}\right)}{\left(64 \pi / 3+\eta^{2}\right)^{2}},
$$

where the modified similarity coordinate $\eta=R /\left(X+X_{O}\right)$ also incorporates a dilatation associated with the apparent origin. The solution satisfies the boundary conditions given in (3.4) together with the additional integral constraint (3.6).

The value of the apparent origin can be calculated from continuity considerations. Thus, integrating (3.5) gives the increasing jet volume flux

$$
\int_{0}^{\infty} 2 \pi R U_{j} \mathrm{~d} R=g_{o}+8 \pi\left(X+\int_{0}^{X}(\Phi / 4-1) \mathrm{d} X\right)
$$

which can be evaluated at $X \gg 1$, using (3.7), to yield

$$
8 \pi\left(X+X_{O}\right)=g_{o}+8 \pi\left(X+\int_{0}^{\infty}(\Phi / 4-1) \mathrm{d} X\right)
$$

and then

$$
X_{O}=\int_{0}^{\infty}(\Phi / 4-1) \mathrm{d} X+\frac{g_{o}}{8 \pi} .
$$

The value of $X_{O}$ is seen to depend on the shape of the initial velocity distribution through the value of the initial volume flux $g_{o}$ and also through the distribution of entrainment rate $\Phi(X)$. Sample values of $X_{O}$ are 0.130 for the uniform jet and 0.095 for the parabolic velocity profile.

The boundary-layer approximation presented here could be extended to higher 
orders by accounting for smaller terms to determine, for instance, the variations of pressure that occur across the jet. These can be computed by integrating the radial component of the momentum equation

$$
\frac{\partial P_{j}}{\partial R}=-U_{j} \frac{\partial V_{j}}{\partial X}-V_{j} \frac{\partial V_{j}}{\partial R}+\frac{\partial}{\partial R}\left(\frac{1}{R} \frac{\partial\left(R V_{j}\right)}{\partial R}\right),
$$

in which pressure differences $P_{j}$ are scaled with its characteristic value $\rho u_{j}^{2} / R e_{j}^{2}$. The total pressure jump across the jet, $\delta P_{j}(X)=P_{j}(R=0, X)-P_{j}(R=\infty, X)$, was computed for uniform and Poiseuille inlet velocity profiles. The resulting distributions are shown in figure 2, along with their associated values for $X \gg 1$ and for $X \ll 1$. In the Schlichting region corresponding to $X \gg 1$, the presure increment simplifies to $\delta P_{j}=[3 /(8 \pi)]\left(X+X_{O}\right)^{-2}$, as can be obtained by integrating (3.11) with use made of the asymptotic velocity profiles (3.7). On the other hand, the solution for the mixing layers at $X \ll 1$ yields $\delta P_{j}=\left(G_{\infty}^{2} U_{i} / 4\right) X^{-1}$ for the uniform velocity profile $U_{i}=\pi^{-1 / 2}$ and $\delta P_{j}=(8 / 27) G_{\infty}^{2} A^{2 / 3} X^{-2 / 3}$ for the Goldstein mixing layer that forms otherwise, where the velocity gradient $A$ takes the value $A=2(3 / \pi)^{1 / 2}$ for the Poiseuille velocity profile.

The problem of finding the pressure distribution in the jet is in principle coupled to the solution in the outer region $O$, which determines the boundary value for $P_{j}$ as $R \rightarrow \infty$. When $R e_{j}^{2} \varepsilon^{4} \ll 1$ the analysis is simpler, in that the pressure variations in $\mathrm{O}$, of order $\rho \varepsilon^{4} u_{j}^{2}$, are smaller than those occurring across the jet, of order $\rho u_{j}^{2} / R e_{j}^{2}$, and can be consequently neglected when integrating (3.11). Differentiating the resulting value of $\delta P_{j}$ determines the axial pressure gradient along the axis, which takes for instance the value

$$
\frac{\partial P_{j}}{\partial X}=-\frac{3 /(4 \pi)}{\left(X+X_{O}\right)^{3}}
$$

for $X \gg 1$, a result to be used later.

The value of $\delta P_{j}$ calculated above increases as the inlet is approached, so that at distances from the jet exit of the order of the pipe radius $\varepsilon a$, the pressure jump across the mixing layer becomes of order $\rho u_{j}^{2} / R e_{j}$ for the initially uniform velocity profile and of order $\rho u_{j}^{2} / R e_{j}^{4 / 3}$ for a non-uniform velocity profile. These pressure increments will also be found at distances of order $\varepsilon a$ upstream from the jet exit, inducing small changes in the axial velocity of order $u_{j} / R e_{j}$ for the uniform velocity profile and smaller changes of order $u_{j} / R e_{j}^{4 / 3}$ otherwise. The calculation of these perturbations at the mouth of the pipe requires the solution of an elliptic problem, which we do not carry out in this paper because these perturbations are small, and do not influence the flow at the pipe exit at the leading order considered here. Similarly, the pressure differences found in regions $\mathrm{O}, \mathrm{M}$ and $\mathrm{T}$ are also much smaller than $\rho u_{j}^{2}$. Therefore, in confined jets with $\varepsilon \ll 1$ the initial velocity profile $u_{i}$ at the pipe exit, which is assumed to be a known boundary condition in our analysis, can be calculated without accounting for pressure changes in the recirculating flow by analysing the flow in the pipe with the pressure at its exit assumed to be equal to the pressure at the duct outlet when this is downstream, and not too far, from region $\mathrm{T}$.

\section{Fluid motion outside the jet}

As previously anticipated, the fluid motion in region $\mathrm{O}$, in the vicinity of the backstep, is induced by the jet entrainment, and it depends on the effective Reynolds number $R e_{a}=R e_{j} \varepsilon$. Adequate variables of order unity to describe the flow field 
at distances of order $l_{j}=\operatorname{Re}_{a} a$ from the backstep, where $u \sim \varepsilon^{2} u_{j}, v \sim v / a$, and $p \sim \rho \varepsilon^{4} u_{j}^{2}$, are $X=x / \varepsilon, U_{o}=u / \varepsilon, V_{o}=v$ and $P=p / \varepsilon^{2}$. Introducing these variables into (2.1)-(2.3) gives

$$
\begin{gathered}
\frac{\partial U_{o}}{\partial X}+\frac{1}{r} \frac{\partial\left(r V_{o}\right)}{\partial r}=0 \\
U_{o} \frac{\partial U_{o}}{\partial X}+V_{o} \frac{\partial U_{o}}{\partial r}=-\frac{\partial P}{\partial X}+\frac{1}{r} \frac{\partial}{\partial r}\left(r \frac{\partial U_{o}}{\partial r}\right)+\frac{1}{R e_{a}^{2}} \frac{\partial^{2} U_{o}}{\partial X^{2}} \\
\frac{1}{R e_{a}^{2}}\left(U_{o} \frac{\partial V_{o}}{\partial X}+V_{o} \frac{\partial V_{o}}{\partial r}\right)=-\frac{\partial P}{\partial r}+\frac{1}{R e_{a}^{2}} \frac{\partial}{\partial r}\left(\frac{1}{r} \frac{\partial\left(r V_{o}\right)}{\partial r}\right)+\frac{1}{R e_{a}^{4}} \frac{\partial^{2} V_{o}}{\partial X^{2}} .
\end{gathered}
$$

Appropriate boundary conditions for $X>0$ are

$$
r=1: \begin{cases}U_{o}=V_{o}=0 \\ \partial U_{o} / \partial r=V_{o}=0 & \text { (non-slip flow) }\end{cases}
$$

Also, the velocity near the axis must match with that in the jet for $R \gg 1$,

$$
r \rightarrow 0: \quad r V_{o}=-\Phi(X), \quad r \frac{\partial U_{o}}{\partial r}=-\frac{1}{R e_{a}^{2}} \frac{\mathrm{d} \Phi}{\mathrm{d} X} .
$$

At the backstep wall $(0<r \leqslant 1)$, the solution must satisfy the non-slip condition

$$
X=0: \quad U_{o}=V_{o}=0 .
$$

To write the remaining boundary conditions one needs to study the solution to (4.1)(4.3) as $X \rightarrow \infty$, where the entrainment rate reaches the constant value $\Phi(X)=4$, and where the solution in $\mathrm{O}$ matches with that of the main region $\mathrm{M}$.

\subsection{Asymptotic solution for $X \gg 1$}

At distances $X \gg 1$ where $\Phi(X)=4$, equations (4.1)-(4.5) have an exact self-similar solution, with the radial velocity independent of $X$, while the axial velocity and the pressure gradient both increase linearly with distance. Introducing the self-similar variables

$$
X \gg 1: \quad U_{o}=\left[X+X_{O}-g_{o} /(8 \pi)\right] U(r), \quad V_{o}=V(r),
$$

and $\mathrm{d} P / \mathrm{d} X=\left[X+X_{O}-g_{o} /(8 \pi)\right] \Lambda$ into (4.1) and (4.2) gives

$$
\begin{gathered}
U+(1 / r)(r V)_{r}=0 \\
U_{r r}+U_{r}(-V+(1 / r))-U^{2}=\Lambda,
\end{gathered}
$$

to be integrated with boundary conditions $r V=-4$ at $r=0$. For the non-slip solution, the boundary conditions at $r=1$ reduce to $U=V=0$, while $U_{r}=V=0$ must be used for slip flow. In the notation, the subindex $r$ denotes a derivative in the radial direction. The boundary conditions at $r=1$ correspond to those given in (2.7) and (2.8), while the boundary condition at $r=0$ follows from matching the entrainment rate (4.5) evaluated at $X \gg 1$. The translation $X_{O}-g_{o} /(8 \pi)=\int_{0}^{\infty}(\Phi / 4-1) \mathrm{d} X$ employed in the expansions for $U_{o}$ and $\mathrm{d} P / \mathrm{d} X$ guarantees that the total volume flux across the pipe equals $\varepsilon g_{o}$ in agreeement with (2.10), as can be seen by adding (3.8) to the outer volume flux $\int_{0}^{1} 2 \pi r U_{o} \mathrm{~d} r=-8 \pi\left(X+X_{O}\right)+g_{o}$.

For slip flow, the above problem can be integrated exactly to give

$$
U=-8, \quad V=-4\left(1-r^{2}\right) / r, \quad \Lambda=-64 .
$$

On the other hand, the solution for non-slip flow is facilitated by writing the problem 

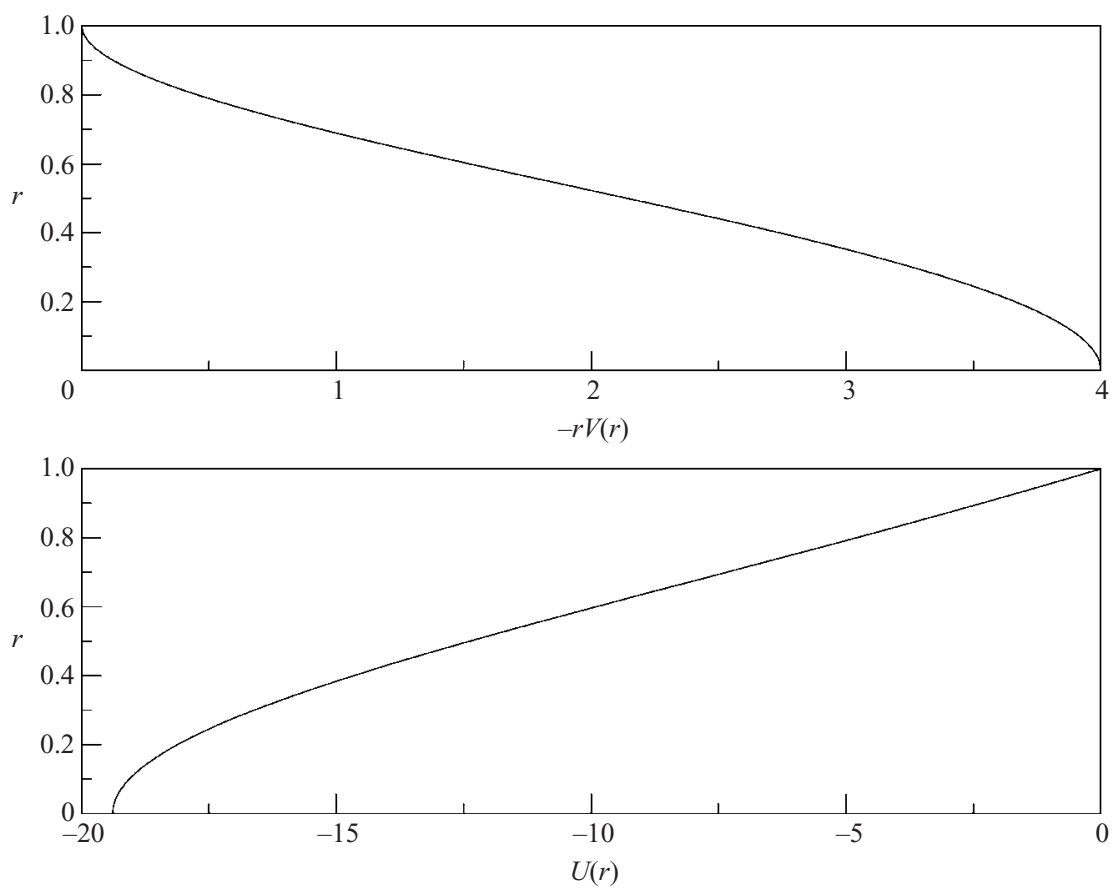

Figure 3. The radial variation of the functions $U(r)$ and $F=-r V$.

in terms of the stream function $\psi(r, X)=\left[X+X_{O}-g_{o} /(8 \pi)\right] F(r)$. Substituing $U=F_{r} / r$ and $V=-F / r$ in (4.9) yields

$$
F_{r r r}+F_{r r}(F-1) / r+F_{r}\left(1-r F_{r}-F\right) / r^{2}=\Lambda r,
$$

to be integrated with boundary conditions

$$
F(1)=F_{r}(1)=0, \quad F(0)=4 .
$$

Integration of the above problem by a shooting method determines the function $F$ as well as the unknown constant $\Lambda=12.258$. Profiles of $F=-r V$ and $U=F_{r} / r$ are displayed in figure 3 . The integrations were initiated at $r \ll 1$, where the function $F$ depends on three unknown constants according to $F=4+\Lambda r^{4} / 48+A_{1} r^{2}+A_{2}+A_{3} r^{-2}$. The two constants $A_{2}=A_{3}=0$ must be chosen to satisfy the boundary condition $F(0)=4$, yielding $F=4+\Lambda r^{4} / 48+A_{1} r^{2}$ as the starting solution at $0<r \ll 1$. An embedded Runge-Kutta-Fehlberg method of fourth and fifth order was employed in the numerical integrations, with the shooting constants $A_{1}$ and $A$ being corrected to satisfy the two conditions $F=F_{r}=0$ at $r=1$ via a Newton-Raphson formula. A value $A_{1}=-9.695$ was found for the solution with $\Lambda=12.258$ shown in figure 3 .

It is worth mentioning that the solution to (4.11) is not unique; a second solution, corresponding to $\Lambda=-387.438$ and $A_{1}=-33.976$, also exists. Unlike the solution in figure 3 , for which the velocity $U$ is negative everywhere, the axial velocity of the second solution vanishes at an intermediate radial location and becomes positive near the wall. Multiple solutions also appear in the solution with slip flow: integration of (4.11) with $F_{r r}=F_{r}\left(U_{r}=0\right)$ replacing the non-slip condition $F_{r}=0$ at $r=1$ gives, in addition to the uniform velocity profile $U=-8$ previously discussed, a second solution for $\Lambda=-194.690$ and $A_{1}=-25.244$ with positive $U$ near the wall. To test the validity of these additional solutions as intermediate asymptotic profiles 
corresponding to $X \gg 1$ and $x \ll 1$, their associated velocity profiles were employed in the boundary condition for integration of the flow in region $M$. The resulting integration showed profiles that evolved rapidly to recover those corresponding to figure 3 for non-slip flow and those given in (4.10) for slip flow, only two or three grid points away from the boundary. This outcome indicates that these additional solutions of (4.11) are irrelevant; although they are similarity solutions of the flow in $\mathrm{O}$ for $X \gg 1$, they do not satisfy the matching condition with the solution in $\mathrm{M}$, and can be therefore discarded in the following development.

\subsection{Leading-order solution in $O$}

The Navier-Stokes equations (4.1)-(4.3) were integrated with boundary conditions (4.4)-(4.7) for different values of the parameter $R e_{a}$ and with entrainment-rate laws $\Phi(X)$ corresponding to both uniform and Poiseuille inlet velocity profiles. The results shown in figures 4 and 5 correspond to a uniform inlet profile with non-slip flow at $r=1$. The equations were solved with the SIMPLE algorithm introduced by Patankar $\&$ Spalding (1972) and Patankar (1980). The problem was formulated as unsteady by including false time derivatives in the two components of the momentum equation, and was integrated in time until the steady state was reached. A three-level fully implicit scheme in time and second-order centred schemes for the spatial derivatives were used for the discretization of the momentum equations. These were solved in every time step with an alternating direction implicit (ADI) procedure. The singular behaviour of the scaled radial velocity $V_{o}$ near the axis was avoided in this problem by replacing the variable $V_{o}$ with $\left(r V_{o}\right)$. The graphs shown correspond to a minimum radius of the integration equal to 0.01 . No significant differences were observed in the results obtained with other values (e.g. 0.02, 0.005). Also, the numerical integrations were performed with the boundary condition as $X \rightarrow \infty$ evaluated at different locations $X \gg 1$ to guarantee that the results are independent of this selection. In particular, for the integrations shown here the profiles (4.7) were evaluated at $X=5$.

Figure $4(a, b)$ exhibits streamlines corresponding to $R e_{a}=1.0$ and 2.5. As expected, the jet entrainment causes the resulting streamlines to deflect towards the axis, except near the corner, where a recirculating eddy emerges. With the streamfunction $\Psi$ defined in the usual manner with $\Psi=0$ at the wall, the streamlines ending at the axis correspond to equal increments $\delta \Psi=0.25$ from this value, while for the slow recirculating flow the spacing of the streamlines is $\delta \Psi=10^{-4}$. According to Moffatt (1964), the non-slender recirculating eddy must be followed by an infinite series of Stokes corner eddies of decaying size and strength, but this local detail of the flow pattern could not be resolved in our computation.

Figure 5 represents the evolution with distance of the scaled pressure gradient $\partial P / \partial X$ along the wall. The pressure gradient, which increases linearly with distance for $X \gg 1$, becomes negative at an intermediate location, then reaches a minimum value and eventually becomes positive again as the corner is approached. The comparison of figures 4 and 5 reveals that the location of minimum pressure gradient coincides approximately with the reattachment point of the streamline bounding the recirculating eddy.

Note that the structure of the flow field near the corner does not change significantly with $R e_{a}$, although this outcome is not apparent in figure 4 because of the scale used for the coordinate $X$. To compare the different non-slender eddies, it is more appropriate to use the radius $a$ to scale both the radial and axial coordinate, as is done in figure 6 to be discussed below.

Note also that the results obtained with an entrainment rate $\Phi(X)$ corresponding to 

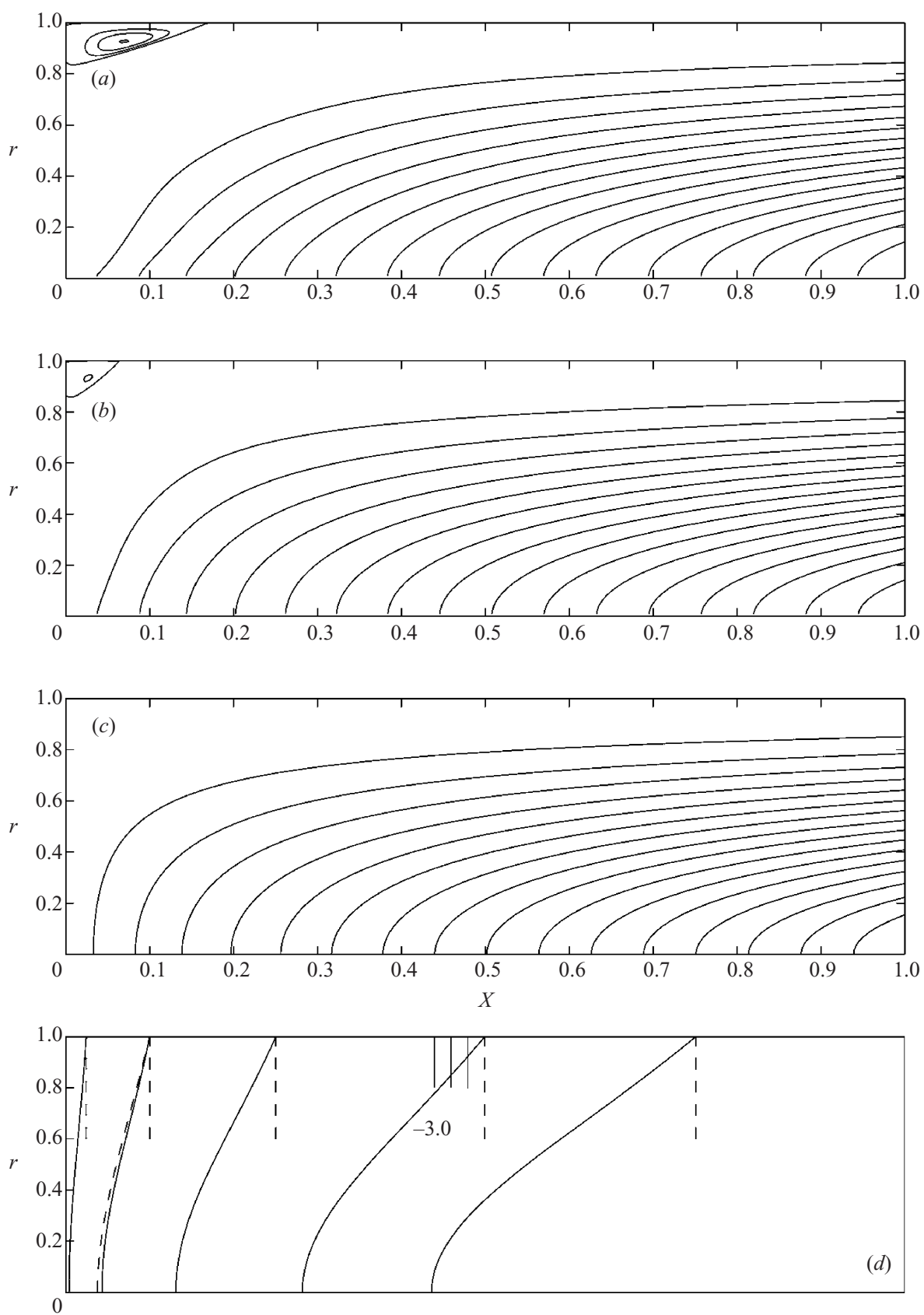

FIGURE 4. Solution in the outer region $O$ with non-slip flow at $r=1$ and uniform inlet velocity profile for $(a) R e_{a}=1.0,(b) R e_{a}=2.5$, and (c) $R e_{a}=\infty$ (BL). Streamlines ending at the axis correspond to increments $\delta \Psi=0.25$ from the $\Psi=0$ value at the wall, while streamlines of recirculating fluid correspond to decrements $\delta \Psi=-10^{-4}$ from this same value. $(d)$ Velocity profiles for the boundary-layer results are given at $X=(0.025,0.1,0.25,0.5,0.75)$ with the velocity scale indicated at $X=0.5$ and with the asymptotic profile $U_{o}=\left[X+X_{O}-g_{o} /(8 \pi)\right] U$ at $X=0.1$ (dashed line) being included for comparison. 


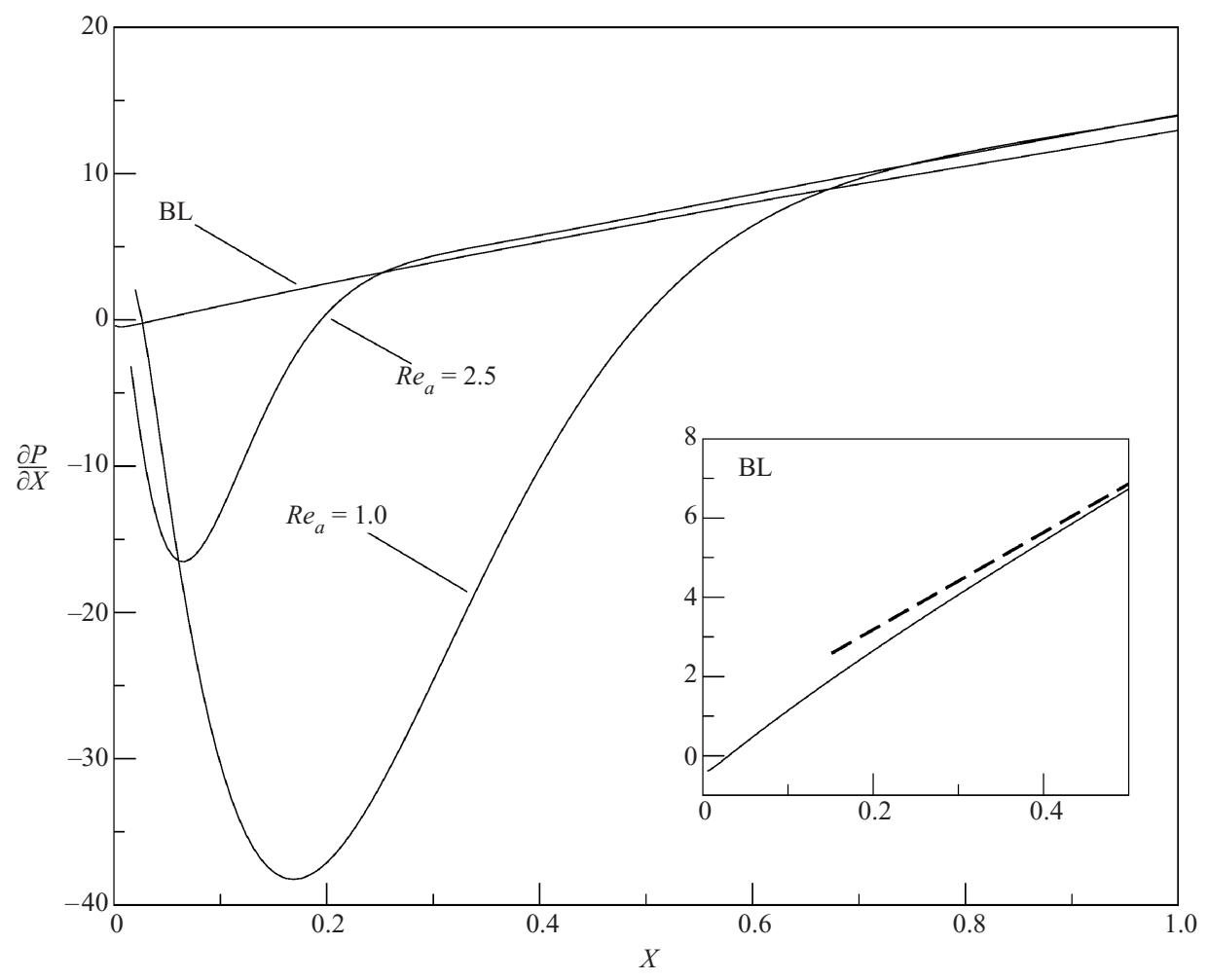

FIGURE 5. The evolution of the axial pressure gradient $\partial P / \partial X$ at the wall obtained in region $\mathrm{O}$ with non-slip flow at $r=1$ and uniform inlet velocity profile for $R e_{a}=1.0, R e_{a}=2.5$, and $R e_{a}=\infty$. The inset shows a magnification of the pressure gradient for $R e_{a}=\infty$, with the dashed line representing its asymptotic behaviour at $X \gg 1$.

a Poiseuille profile change only marginally from those exhibited above for the uniform inlet profile. In particular, the streamlines of the recirculating eddy and the pressure field found at the corner are very similar in both cases. For instance, the pressure gradient along the wall for $R e_{a}=2.5$, which reaches a minimum $\partial P / \partial X \simeq-16.53$ at $X \simeq 0.066$ in figure 5, with Poiseuille entrainment exhibits a minimum value $\partial P / \partial X \simeq-14.79$ at $X \simeq 0.061$. Significant quantitative changes are only found near the origin, where differences in the entrainment rate are more strongly felt, thereby modifying the velocity field in agreement with (4.5). For the case of slip flow at the wall, the differences with the results in figures 4 and 5 are more pronounced, and affect in particular the flow pattern near the corner, where there is no recirculating eddy.

\subsubsection{The limit of large Reynolds numbers, $R e_{a} \gg 1$}

In this limiting case, the boundary-layer approximation can be used to describe the flow in $\mathrm{O}$ at distances from the backstep of order $l_{j}=R e_{a} a$, as can be seen by taking the limit $R e_{a} \rightarrow \infty$ in (4.1)-(4.3). The corresponding equations, obtained by replacing (4.3) with $\partial P / \partial r=0$ and by neglecting the last term in (4.2), can be integrated with boundary conditions (4.4) and (4.5) and with an initial velocity profile at $X \gg 1$ given in (4.7). Since the function $U(r)$ shown in figure 3 is negative everywhere, the flow is directed towards the backstep in this outer region for $X \gg 1$, and the resulting problem becomes parabolic in decreasing values of $X$. Integration can be performed 
by marching from $X \gg 1$ towards the backstep, provided $U_{o}$ remains negative across the pipe, a result that was verified in the computations.

When slip flow is considered at $r=1$, the boundary-layer problem can be integrated exactly to give

$$
U_{o}=-2 \int_{0}^{X} \Phi \mathrm{d} X, \quad V_{o}=-\Phi\left(1-r^{2}\right) / r, \quad \frac{\mathrm{d} P}{\mathrm{~d} X}=-4 \Phi \int_{0}^{X} \Phi \mathrm{d} X .
$$

Numerical integration is required to solve the problem however when non-slip boundary conditions at $r=1$ are employed. The procedure was implemented with an implicit numerical scheme. Although the presence of a singularity in $\Phi$ at $X=0$ complicates the computation of the solution in the inmediate vicinity of the backstep, the integration could be extended to values of $X$ as small as $X=2 \times 10^{-3}$. Entrainment rates $\Phi(X)$ corresponding to both uniform and parabolic jets were considered. Since only backflow (negative values of $U_{o}$ ) was found in the computations, the parabolicity of the problem and the validity of the results obtained up to the smallest value of $X$ computed is guaranteed.

Results corresponding to a uniform inlet velocity profile are shown in figures 4 and 5. Figure 4 represents streamlines, with the plot $(d)$ exhibiting the associated velocity profiles $U_{o}$ at different axial locations. Following the transition of the entrainment rate to the Schlichting solution observed in figure 2 , the axial velocity profiles are seen to evolve rapidly towards the asymptotic solution $U_{o}=\left[X+X_{O}-g_{o} /(8 \pi)\right] U$, as shown in the figure by comparing at $X=0.1$ the asymptotic velocity profile with that obtained numerically. On the other hand, figure 5 represents the evolution with distance of the normalized pressure gradient $\mathrm{d} P / \mathrm{d} X$, along with its asymptotic behaviour $\mathrm{d} P / \mathrm{d} X=\left[X+X_{O}-g_{o} /(8 \pi)\right] \Lambda$ corresponding to $X \gg 1$. Although the computation reveals a negative pressure gradient at $X \ll 1$, the associated deceleration was not enough to reverse the backflow in the domain computed.

Note that the boundary-layer approximation can be expected to fail at distances $x \sim a$ from the backstep $\left(X \sim R e_{a}^{-1}\right)$, in a region where $u \sim v \sim R e_{a}^{\alpha} v / a$. For the analysis of this inviscid region of strong streamline deflection, not given here, the rescaled variables $R e_{a} X, R e_{a}^{1-\alpha} U_{o}, R e_{a}^{-\alpha} V_{o}$ and $R e_{a}^{2(1-\alpha)} P$ should be introduced. Boundary conditions for the associated Euler equations must consider the large entrainment rate $\Phi=C X^{-\alpha}$ at the axis as well as the velocity profiles found at the end of the boundary-layer region.

\subsubsection{The limit of small Reynolds numbers, $R e_{a} \ll 1$}

In the opposite limit $R e_{a} \ll 1$, the region of streamline deflection $x \sim a$, where the characteristic velocity components are $u \sim v \sim v / a$, is much larger than the region of jet development. To describe the outer motion, it is therefore convenient to use $a$ and $v / a$ as scales for lengths and velocities. They lead to the rescaled axial coordinate $\hat{X}=x / a=R e_{a} X$ and modified variables $\hat{U}_{o}=u /(v / a)=R e_{a} U_{o}$ and $\hat{P}=p /\left(\rho v^{2} / a^{2}\right)=R e_{a}^{2} P$, to be used to write the conservation equations (4.1)-(4.3) in a form independent of $R e_{a}$. The resulting parameter-free Navier-Stokes equations must be integrated with boundary conditions, obtained by neglecting small terms of order $R e_{a}$ in (4.4)-(4.7), given by

$$
\hat{X}>0: \begin{cases}r=0: & r V_{o}=-4 \\ r=1: & \begin{cases}\hat{U}_{o}=V_{o}=0 \\ \partial \hat{U}_{o} / \partial r=V_{o}=0 & \text { (non-slip flow) }\end{cases} \end{cases}
$$


and

$$
0<r \leqslant 1: \quad \begin{cases}\hat{X}=0: & \hat{U}_{o}=V_{o}=0 \\ \hat{X}=\infty: & \hat{U}_{o}=\hat{X} U(r), \quad V_{o}=V(r) .\end{cases}
$$

The resulting flow field is similar to those given above for finite values of $R e_{a}$. Specifically, in an $r-\hat{X}$ plot the resulting streamline pattern is practically identical to that shown in the $r-X$ plot of figure 4 for $R e_{a}=1$. In this limiting case $R e_{a} \ll 1$, the flow near the origin approaches the similarity Navier-Stokes solution proposed by Schneider (1981). This region of self-similar flow corresponds to distances from the jet entrance much smaller than $a$ and yet much larger than $l_{j}=\operatorname{Re}_{a} a$, so that the entrainment rate at the axis becomes constant.

As previously noted, the flow-field structure at the corner is weakly dependent on the value of $R e_{a}$, an outcome that is investigated in figure 6(a), where the streamlines corresponding to values of the rescaled streamfunction $\hat{\Psi}=\operatorname{Re}_{a} \Psi=$ $\left(0,-10^{-4},-2 \times 10^{-4}\right)$ are plotted for the three rescaled Reynolds numbers $R e_{a}=$ $(0,1.0,2.5)$. The agreement obtained is seen to be excellent for the values of $R e_{a}$ considered. For the axial pressure field at the wall, the results in figure 5 suggest that the difference between the axial pressure gradient and its asymptotic value at $X \gg 1$, $\partial P / \partial X=\left[X+X_{O}-g_{o} /(8 \pi)\right] \Lambda$, scales with $R e_{a}^{-1}$. To further elucidate this issue, we also represent in figure $6(b)$ the variation of $\operatorname{Re}_{a} \delta P=\operatorname{Re}_{a}\left\{\partial P / \partial X-\left[X+X_{O}-g_{o} /(8 \pi)\right] \Lambda\right\}$ along the wall for $\operatorname{Re}_{a}=(1.0,2.5)$ and also for $\operatorname{Re}_{a}=0\left(\operatorname{Re}_{a} \delta P=\partial \hat{P} / \partial \hat{X}-\Lambda \hat{X}\right)$. Again, the agreement found further confirms that the flow pattern at the corner is weakly dependent on $R e_{a}$, so that the solution for $R e_{a}=0$ remains accurate even for moderately large values of $R e_{a}$.

\section{The main recirculating region}

The conservation equations in $\mathrm{M}$ are those given above in (2.1)-(2.3), which reduce, with small relative errors of order $R e_{j}^{-2}$, to the boundary-layer equations

$$
\begin{gathered}
\frac{\partial u}{\partial x}+\frac{1}{r} \frac{\partial(r v)}{\partial r}=0 \\
u \frac{\partial u}{\partial x}+v \frac{\partial u}{\partial r}=-\frac{\mathrm{d} p}{\mathrm{~d} x}+\frac{1}{r} \frac{\partial}{\partial r}\left(r \frac{\partial u}{\partial r}\right) .
\end{gathered}
$$

These equations are to be integrated with an initial velocity profile determined by matching the solution in $\mathrm{M}$ with the solution in regions $\mathrm{J}$ and $\mathrm{O}$. Thus, combining the outer solution for $X \gg 1$ given in (4.7) with the Schlichting solution given in (3.7) yields, with relative errors of order $\varepsilon^{2}$,

$$
u=\left[x+\varepsilon\left(X_{O}-\frac{g_{o}}{8 \pi}\right)\right] U(r)+\frac{512 \pi}{3\left(x+\varepsilon X_{O}\right)}\left(\frac{64 \pi}{3}+\frac{r^{2}}{\left(x+\varepsilon X_{O}\right)^{2}}\right)^{-2}
$$

for the velocity profile at $x=0$. To proceed with the asymptotic analysis, expansions in powers of $\varepsilon$ (or, alternatively, in powers of $R e_{j}^{-1}$, since both procedures are equivalent in the distinguished limit $\operatorname{Re}_{j} \varepsilon \sim 1$ used here) should be introduced for the different flow variables. Solving the resulting problem arising at each order then produces the required solution in a sequential manner. Since the boundary-layer equations (5.1)(5.2) and the associated initial velocity profile (5.3) have relative errors of order $R e_{j}^{-2}$ and $\varepsilon^{2}$, respectively, they can be used to calculate the first two terms in the asymptotic 

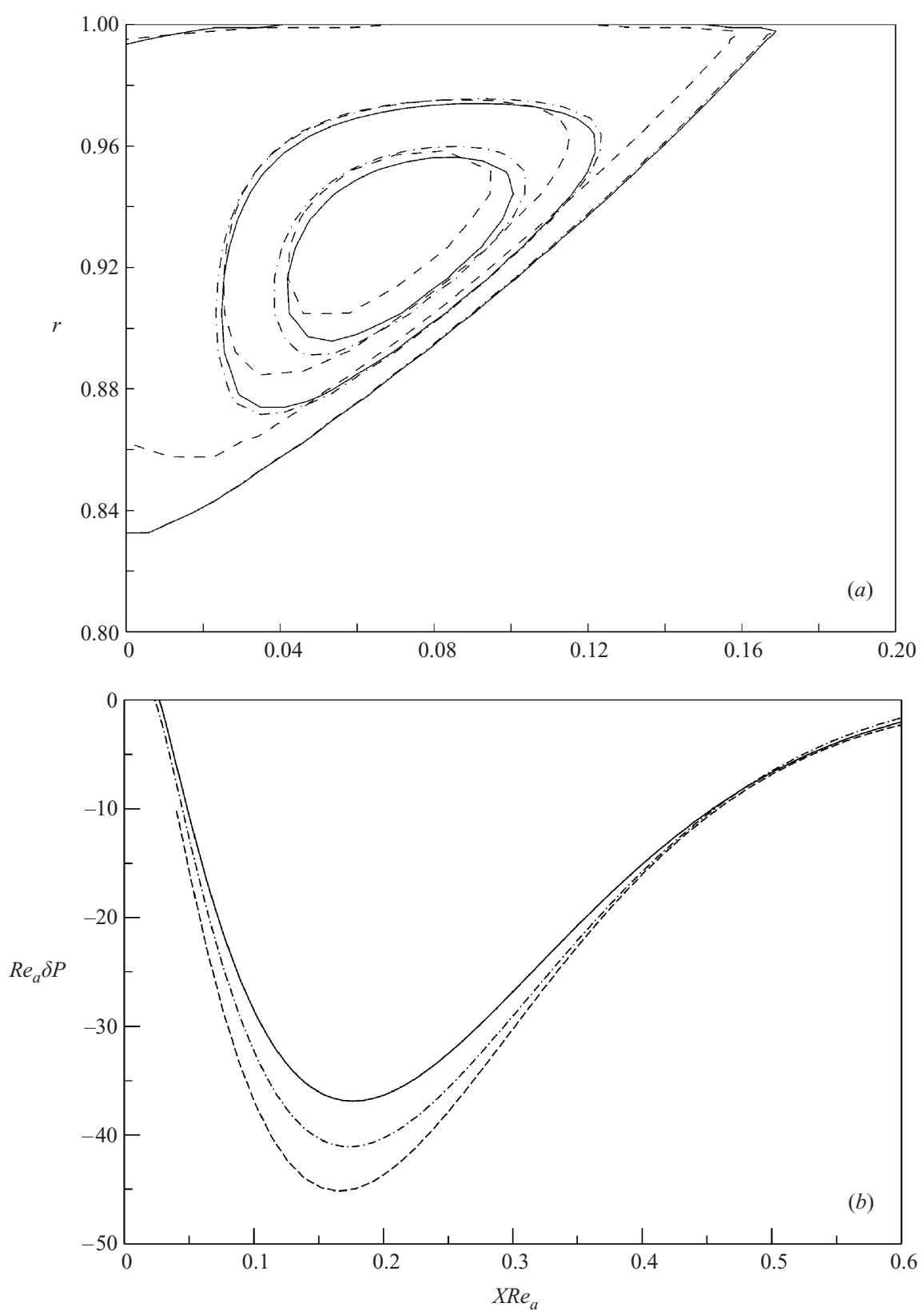

FIGURE 6. (a) Equally spaced streamlines near the corner with $\delta \hat{\Psi}=10^{-4}$ for $R e_{a}=0$ (solid lines), $R e_{a}=1.0$ (dot-dashed lines) and $R e_{a}=2.5$ (dashed lines); $(b)$ the corresponding incremental pressure gradient $\operatorname{Re}_{a} \delta P$ along the wall.

expansion presented below. Details of the finite-volume scheme used to integrate the boundary-layer equations are given in an appendix.

\subsection{Leading-order description}

At leading order, the problem reduces to that of integrating (5.1) and (5.2) with boundary conditions at the axis and on the wall given in (2.6)-(2.8), and with an 

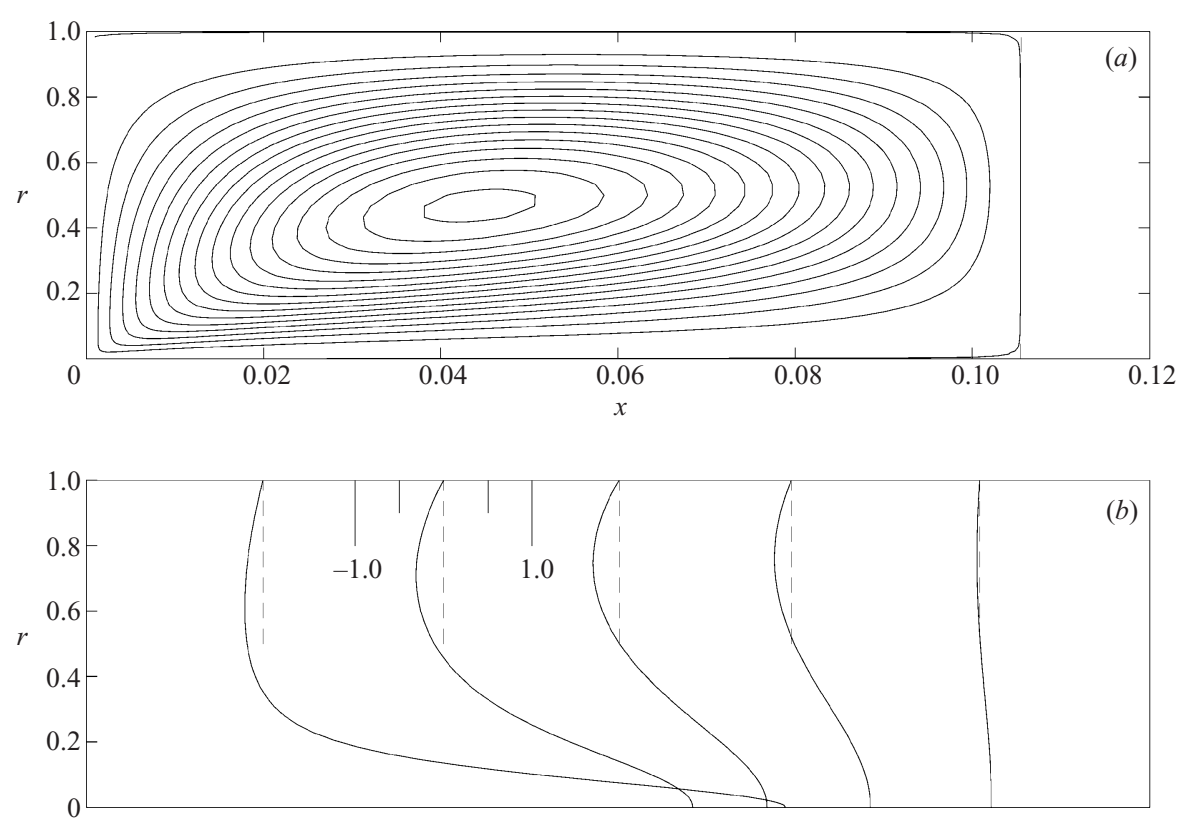

FIGURE 7. The leading-order solution for the confined jet with non-slip flow at $r=1:(a)$ streamlines (the stream function for outermost streamline is $\psi=5 \times 10^{-6}$ and the remaining streamlines correspond to increasing values of $\psi$ from $\psi=5 \times 10^{-3}$ in equal increments $\delta \psi=5 \times 10^{-3}$ ) and (b) axial velocity profiles at $x=(0.02,0.04,0.06,0.08,0.10)$, with the velocity scale indicated for the profile at $x=0.04$.

initial velocity profile

$$
u=x U(r)+\frac{512 \pi}{3 x}\left(\frac{64 \pi}{3}+\frac{r^{2}}{x^{2}}\right)^{-2}
$$

obtained from (5.3) with $\varepsilon=0$. In this leading-order description, the jet acts as a point source of momentum with negligible mass flow rate, yielding a parameter-free problem independent of the shape of the inlet velocity profile. The mass flux and the shape of the inlet velocity profile will enter at the following order, in agreement with (2.10).

Figures 7 and 8 show the streamlines of the resulting flow field with non-slip and slip flow, respectively, along with characteristic axial velocity profiles at different downstream locations. As a result of the zero mass flux associated with the leadingorder description, the axial velocity vanishes at a finite location $x_{s}$ and the flow remains stagnant downstream. For non-slip flow, the eddy extends to $x_{s} \simeq 0.106$, while due to the absence of viscous forces at $r=1$ the eddy length increases to $x_{s} \simeq 0.131$ for slip flow. With the stream function $\psi$ defined in the usual manner with $\psi=0$ along the axis, the maximum value obtained at the eddy centre was seen to be $\psi=0.0816$ for non-slip flow and $\psi=0.1057$ for slip flow. The outermost streamline depicted in both plots corresponds to $\psi=5 \times 10^{-6}$, whereas values of the stream function for the remaining streamlines increase from $\psi=5 \times 10^{-3}$ in equal increments $\delta \psi=5 \times 10^{-3}$ (for non-slip flow) and $\delta \psi=10^{-2}$ (for slip flow).

Note that the eddy length $x_{s} \simeq 0.106$ calculated here differs significantly from that obtained in the numerical integrations of axysimmetric sudden expansions reported in Acrivos \& Schrader (1982). Their prediction for the eddy length when $\varepsilon \rightarrow 0$ can 

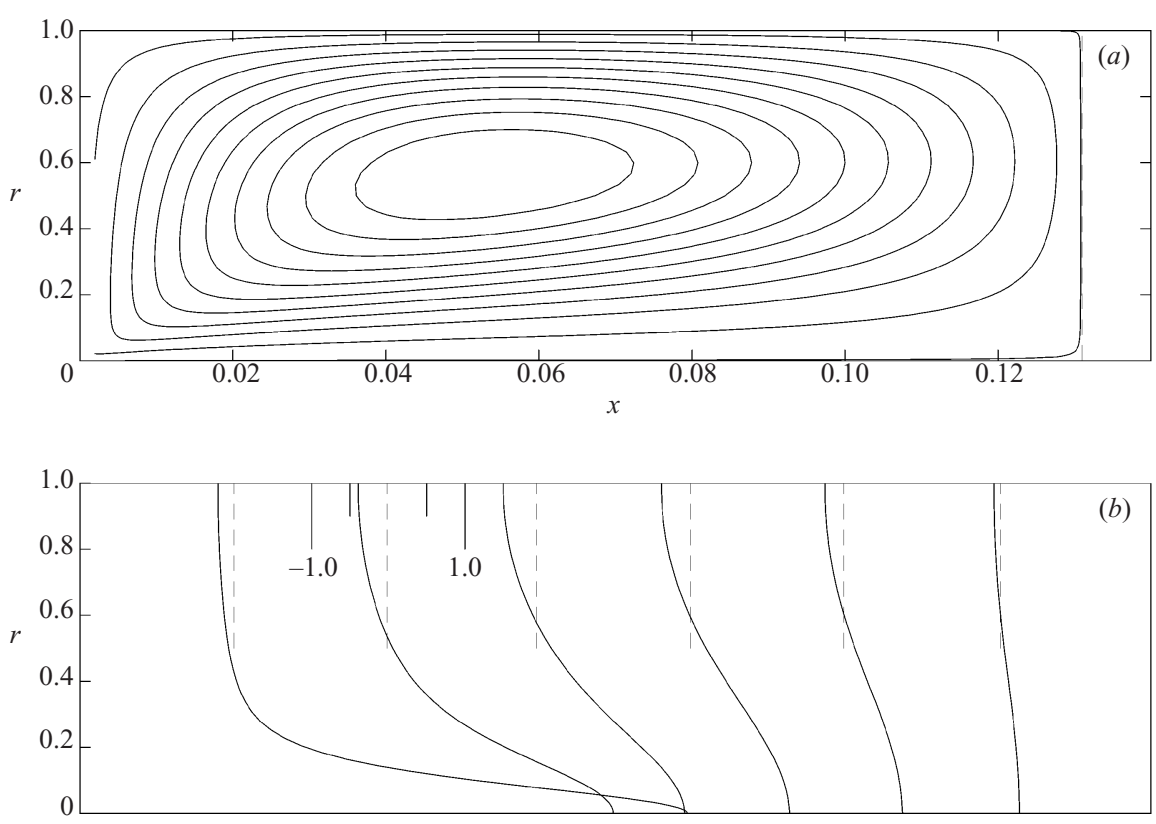

FIGURE 8. The leading-order solution for the confined jet with slip flow at $r=1:(a)$ streamlines (the stream function for outermost streamline is $\psi=5 \times 10^{-6}$ and the remaining streamlines correspond to increasing values of $\psi$ from $\psi=5 \times 10^{-3}$ in equal increments $\delta \psi=10^{-2}$ ) and $(b)$ axial velocity profiles at $x=(0.02,0.04,0.06,0.08,0.10,0.12)$, with the velocity scale indicated for the profile at $x=0.04$.

be written as $x_{s} \simeq 0.119$ in terms of the axial coordinate used here. This discrepancy may be explained by recalling that Acrivos \& Schrader (1982) treated the case $\varepsilon \ll 1$ only marginally; they were in fact interested in expansion ratios of order unity or smaller. Perhaps their numerical integrations were not extended to cover sufficiently small values of $\varepsilon$, or their numerical scheme was not adapted well to describe the singular behaviour associated with the jet in the limit $\varepsilon \rightarrow 0$.

Figure $9(a)$ shows the pressure gradient, which is compared with the asymptotic prediction $\mathrm{d} p / \mathrm{d} x=x \Lambda$ associated with (5.4). The pressure gradient with non-slip flow increases from a zero initial value at the entrance to reach a maximum value $\mathrm{d} p / \mathrm{d} x \simeq 10.49$ at $x=0.061$. For $x>0.061, \mathrm{~d} p / \mathrm{d} x$ continuously decreases, vanishing as the eddy rear end is approached. With slip flow at $r=1$, the pressure gradient is initially negative, although it eventually becomes positive, reaching a maximum value $\mathrm{d} p / \mathrm{d} x \simeq 6.077$ at $x=0.080$. The different behaviour can be easily explained in physical terms. With slip flow at $r=1$ the initial pressure gradient is negative as required to inviscidly decelerate the outer flow that approaches the backstep, whereas in the case of non-slip flow the pressure gradient is initially positive to overcome the viscous force exerted by the confining wall.

Integration of the pressure gradient provides the pressure variations from the corner value, which are also shown in the figure. The final value $(p \simeq 0.593$ for non-slip flow and $p \simeq 0.318$ for slip flow) gives the total pressure increment associated with the sudden expansion. Note that the value obtained numerically with slip flow agrees well with the exact value $p=1 / \pi$, determined in this case using the integral form of the momentum equation for the fluid volume occupied by the eddy.

For completeness, figure $9(b)$ also shows the evolution with $x$ of the axial velocity 

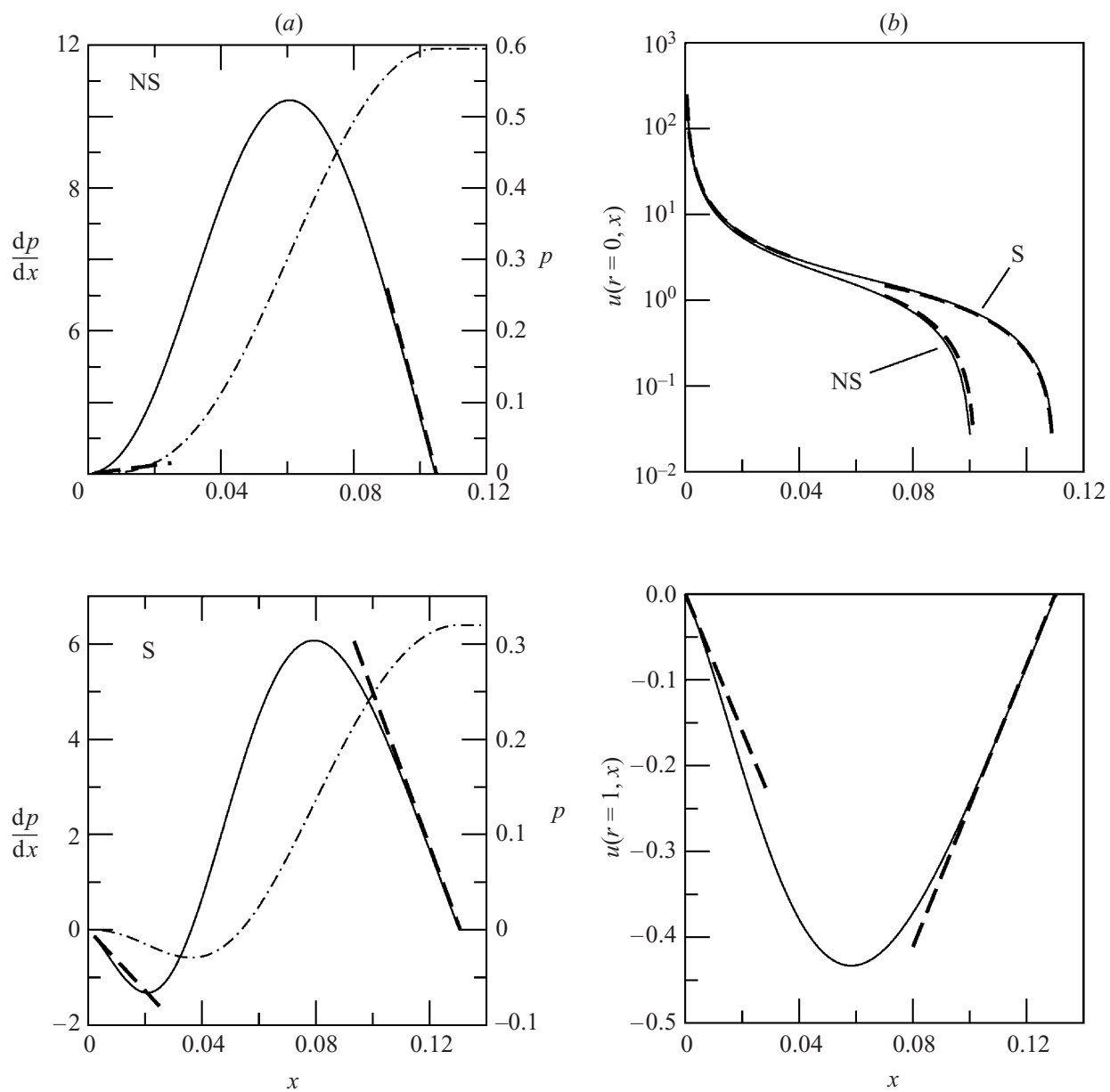

FIGURE 9. (a) The pressure gradients (solid lines) and associated leading-order pressure distributions (dot-dashed lines) corresponding to the confined jet with both non-slip flow (NS) and slip flow (S), and $(b)$ the corresponding distributions of axial velocity at the axis (upper plot) and at the wall (lower plot); dashed lines represent the asymptotic predictions for $x \ll 1$ and for $\left(x_{s}-x\right) \ll 1$.

$u$ along the axis (for both non-slip and slip flow) and also at the wall (for slip flow). The results are compared with the boundary solutions $u=3 /(8 \pi x)$ at $r=0$ and $u=-8 x$ at $r=1$ obtained from (5.4) for $x \ll 1$. The velocity along the axis is seen to evolve from the large jet value to the vanishing values found as the stagnation plane, $x=x_{s}$, is approached, showing a disparity of scales that is exhibited in the plot by using a logarithmic scale for $u$. The negative velocity at the slip wall is seen to reach a minimum value $u=-0.433$ at a location $x=0.0582$ upstream from the point of maximum pressure gradient.

\subsection{First-order corrections}

The solution described above represents at leading order the flow field in region $\mathrm{M}$. The first-order correction, of order $\varepsilon$, must satisfy the linearized form of the boundarylayer equations (5.1) and (5.2) subject to an initial condition determined by linearizing (5.3) about the leading-order solution. Combining the leading-order results with the correction in a two-term expansion would then provide a description for the flow field 
in the main region $\mathrm{M}$ with small relative errors of order $\varepsilon^{2}$. This expansion fails at $x=x_{s}$, where the correction for the axial velocity is seen to develop a logarithmic singularity proportional to $\ln \left(x_{s}-x\right)$ as the leading-order solution vanishes. As seen below in (6.7), the description of the transition region towards the final downstream solution must account for the presence of this singularity.

Instead of following this standard procedure, we choose here to integrate (5.1) and (5.2) with the initial velocity profile given in (5.3). This alternative method yields the solution in $\mathrm{M}$ with relative errors of order $\varepsilon^{2}$, just like the two-term expansion previously discussed. The main advantage is that the resulting solution does not become singular at $x=x_{s}$, and reproduces the asymptotic flow found downstream, thereby providing results that can be easily compared with integrations of the full Navier-Stokes problem (2.1)-(2.9). Nevertheless, the procedure proposed is not expected to describe accurately the solution in the boundary region $T$, which is considered separately below.

The results obtained with the initial velocity profile (5.3) are similar to the leadingorder results previously described. The main differences stem from the non-zero mass flow rate (2.10), which causes the flow downstream to approach either the Poiseuille solution with a negative constant value of the pressure gradient (if non-slip flow is considered) or a uniform velocity profile with zero pressure gradient (for slip flow). Streamlines corresponding to the case $\varepsilon=0.05$ obtained for a Poiseuille inlet profile with non-slip outer wall are given in figure 10(e). The results of the integration, notably the resulting eddy length, were seen to be quite independent of the initial location used to evaluate (5.3) provided $1 \gg x \gtrsim 0.1 \varepsilon(x=0.005$ for the integration shown in the figure). As can be seen, the toroidal vortex is very similar to that shown in figure 7 for the case $\varepsilon=0$. In particular, the streamline separating the recirculating flow intersects the wall at a location $x_{f}$ that differs by a small amount $\left(x_{f}-x_{s}\right) / x_{s} \sim \varepsilon$ from the eddy length $x_{s}$ of figure 7 .

The results of the boundary-layer analysis were compared with numerical integrations of the full Navier-Stokes problem formulated in (2.1)-(2.9). As in the integration of the problem (4.1)-(4.6), the SIMPLE algorithm was used. In the computations, (2.9) was replaced with an outflow boundary condition. Streamlines corresponding to values of the Reynolds number ranging from $R e_{j}=20$ to $R e_{j}=500$ are plotted in figure $10(a-d)$. As can be seen, the streamline pattern is very similar for all cases considered. The eddy length predicted by the boundary-layer approximation matches the results of the Navier-Stokes integrations, although larger discrepancies occur as $R e_{j}$ decreases. Note that the corner eddy encountered above in region $\mathrm{O}$ also appears in the Navier-Stokes integrations for the two smaller $R e_{j}$ considered.

Further comparisons between the boundary-layer results and those of the full integrations are given in figure 11. Figure 11(c) shows the evolution of the flow velocity along the axis as obtained with the boundary-layer approximation and with the Navier-Stokes equations for $R e_{j}=50$ and 200. The agreement is very good in both cases, with the curve for $R e_{j}=200$ being practically indistinguishable from that of the boundary-layer results over the range of $x$ considered.

Comparisons of axial pressure gradients obtained for $R e_{j}=50$ and 200 with that obtained from the boundary-layer approach are given in figure $11(a, b)$; plot $(b)$ shows the evolution of $\partial p / \partial x$ at the wall. The boundary-layer approximation reproduces accurately the results for $R e_{j}=200$, whereas somewhat larger discrepancies are seen for the case $R e_{j}=50$. Notice also that as the backstep is approached the pressure gradient at the wall reproduces the behaviour predicted in the outer region O, i.e. it exhibits a minimum value that is more pronounced for smaller values of $R e_{a}=R e_{j} \varepsilon$. 

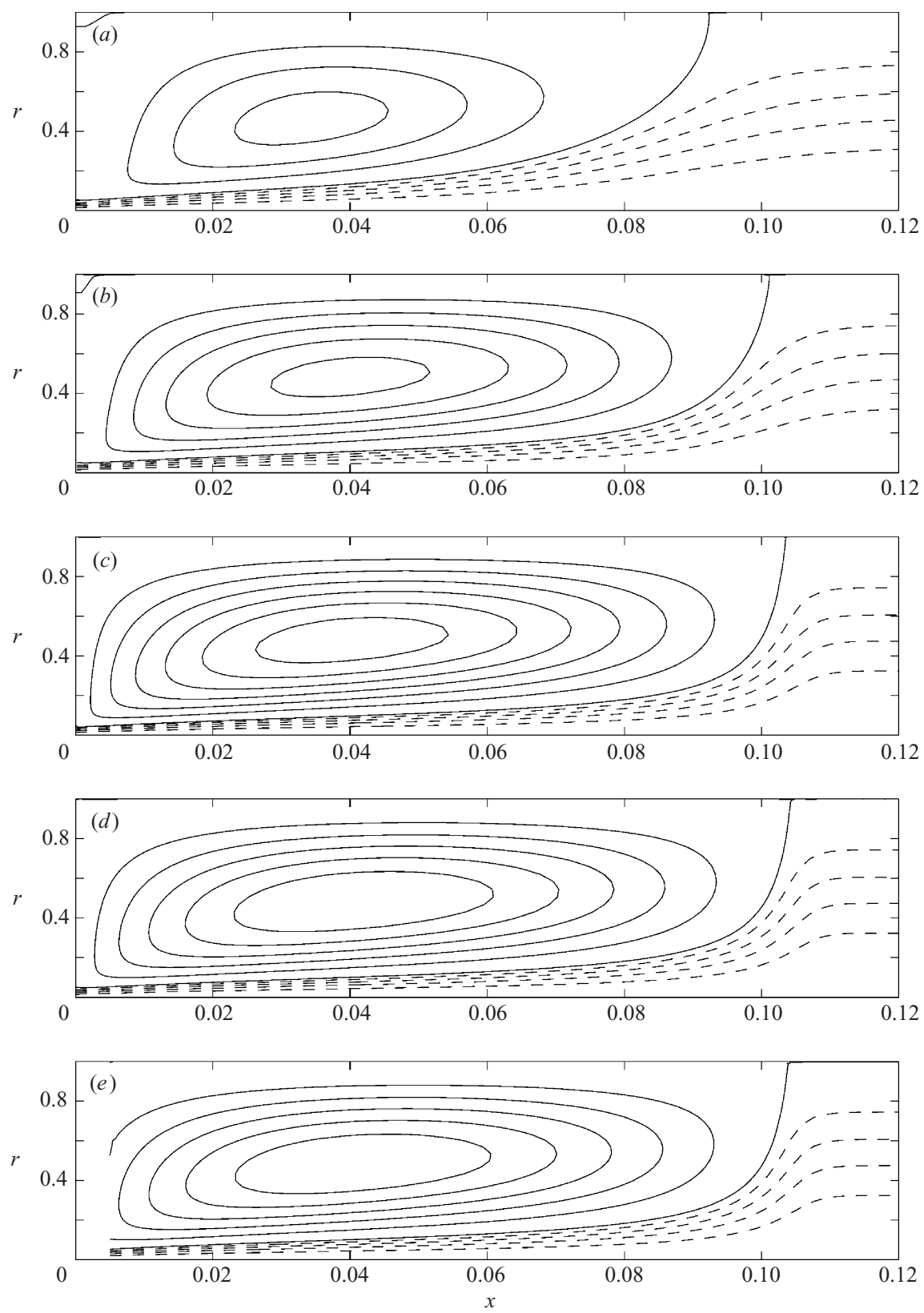

FIGURE 10. Streamlines of the confined jet for $\varepsilon=0.05$ with non-slip flow at $r=1$ and Poiseuille inlet velocity profile as obtained by integrating the boundary-layer equations (5.1) and (5.2) (e) and by integrating the full Navier-Stokes problem (2.1)-(2.9) for different values of the Reynolds number $R e_{j}:(a) R e_{j}=20,(b) R e_{j}=50,(c) R e_{j}=200,(d) R e_{j}=500$. The recirculating streamlines (solid lines) correspond to increments $\delta \psi=g_{o} \varepsilon /(2 \pi)$ from the $\psi=0$ value of the dividing streamline, while streamlines of escaping fluid (dashed lines) correspond to decrements $\delta \psi=-g_{o} \varepsilon /(10 \pi)$ from this same value. 

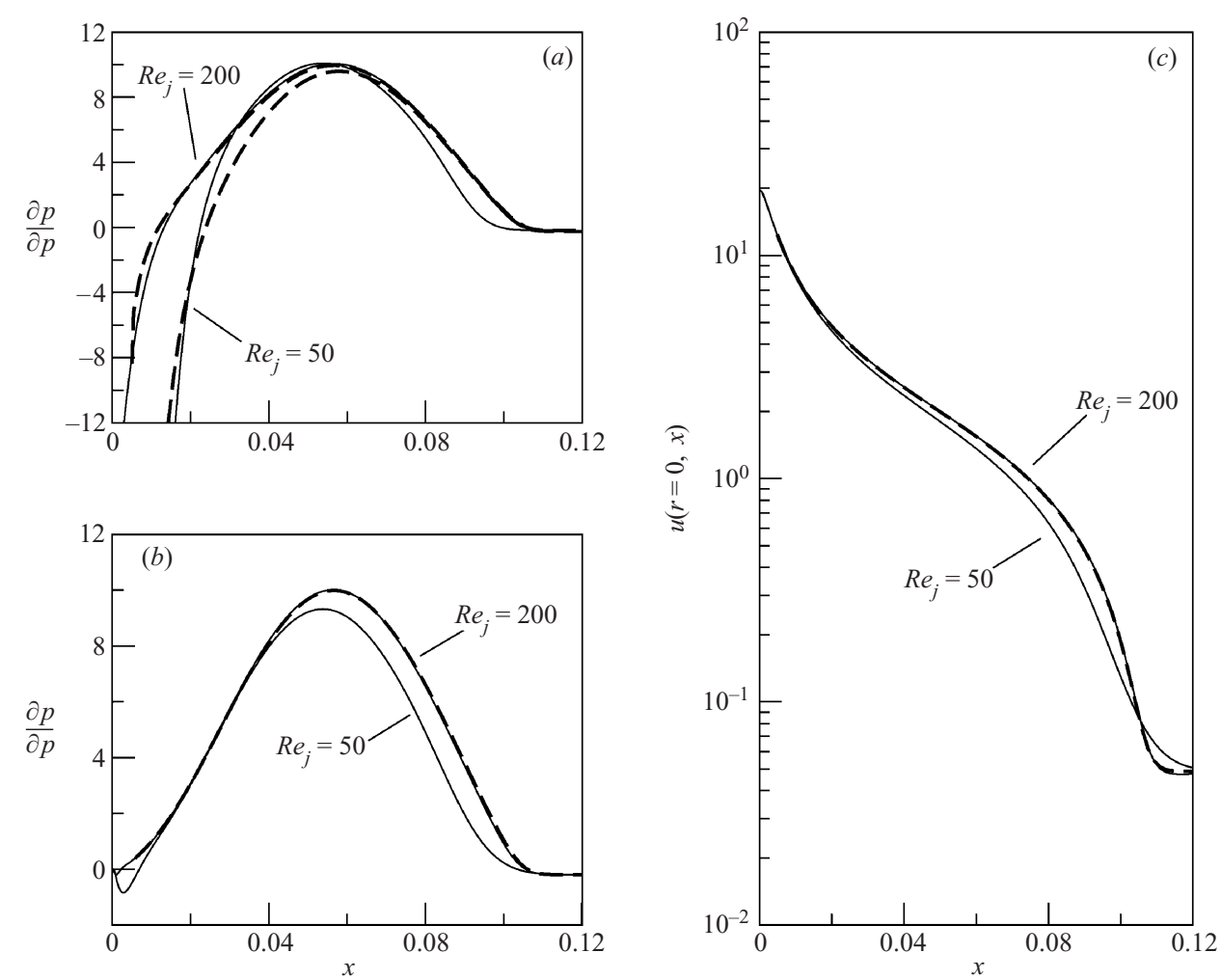

FIGURE 11. The pressure gradients at the wall $(b)$ and at the axis $(a)$, along with the axial velocity distribution along the axis $(c)$ as obtained from the boundary-layer results (dashed lines) and from the full Navier-Stokes problem (solid lines) for $\varepsilon=0.05$ with non-slip flow at $r=1$ and Poiseuille profile at the inlet.

The pressure gradient along the axis is shown in figure 11(a). To account for the presence of the jet, the pressure gradient of the asymptotic analysis is constructed in this case by adding the contribution of Schlichting solution $\partial p / \partial x=-(3 / 4 \pi) R e_{j}^{-2}(x+$ $\left.\varepsilon X_{O}\right)^{-3}$, calculated above in (3.12), to the distribution obtained in region $\mathrm{M}$ from (5.1) and (5.2). The resulting composite expansion for the pressure gradient is seen to describe well the Navier-Stokes results for the two values of $R e_{j}$ considered.

\section{The transition region}

As previously anticipated, the dividing streamline eventually opens up at the rear end of the recirculating region, leading to a downstream region of parallel flow through a short transition region, denoted by $\mathrm{T}$ in the sketch of figure 1 , where $u$ is of order $\varepsilon$. If a non-slip wall is considered, as in the calculation of figure 10, then the pressure gradient, which was positive in the recirculating region, becomes negative somewhere in this transition region, and reaches the Poiseuille value shortly downstream. A uniform stream with zero pressure gradient replaces the Poiseuille solution downstream from the transition region if slip flow is considered at $r=1$.

The description of the flow structure in region $\mathrm{T}$ employes a stretched axial coordinate $\bar{X}=\left(x-x_{e}\right) / \varepsilon$, where $x_{e}$ is a given location within this region. The choice of $x_{e}$ is in principle arbitrary; one could for instance use $x_{e}=x_{s}$ or define $x_{e}$ as the 
location where the pressure reaches its peak value (in non-slip flow configurations). This arbitrariness will be removed when defining the boundary conditions at $\bar{X}=-\infty$. Introducing the coordinate $\bar{X}$ together with the variables $U_{t}=u / \varepsilon, V_{t}=v$ and $\bar{P}=p / \varepsilon^{2}$, reduces $(2.1)-(2.3)$ to

$$
\begin{gathered}
\frac{\partial U_{t}}{\partial \bar{X}}+\frac{1}{r} \frac{\partial\left(r V_{t}\right)}{\partial r}=0 \\
U_{t} \frac{\partial U_{t}}{\partial \bar{X}}+V_{t} \frac{\partial U_{t}}{\partial r}=-\frac{\partial \bar{P}}{\partial \bar{X}}+\frac{1}{r} \frac{\partial}{\partial r}\left(r \frac{\partial U_{t}}{\partial r}\right)+\frac{1}{R e_{a}^{2}} \frac{\partial^{2} U_{t}}{\partial \bar{X}^{2}} \\
\frac{1}{R e_{a}^{2}}\left(U_{t} \frac{\partial V_{t}}{\partial \bar{X}}+V_{t} \frac{\partial V_{t}}{\partial r}\right)=-\frac{\partial \bar{P}}{\partial r}+\frac{1}{R e_{a}^{2}} \frac{\partial}{\partial r}\left(\frac{1}{r} \frac{\partial\left(r V_{t}\right)}{\partial r}\right)+\frac{1}{R e_{a}^{4}} \frac{\partial^{2} V_{t}}{\partial \bar{X}^{2}},
\end{gathered}
$$

to be integrated with boundary conditions

$$
r=0: \quad \partial U_{t} / \partial r=V_{t}=0
$$

and

$$
r=1: \quad \begin{cases}U_{t}=V_{t}=0 \\ \partial U_{t} / \partial r=V_{t}=0 & \text { (non-slip flow) }\end{cases}
$$

The boundary conditions far downstream,

$$
\bar{X} \gg 1: \quad \begin{cases}U_{t}=\left(2 g_{o} / \pi\right)\left(1-r^{2}\right), V_{t}=0 & \text { (non-slip flow) } \\ U_{t}=g_{o} / \pi, V_{t}=0 & \text { (slip flow) }\end{cases}
$$

follow from rewriting (2.9) for the present variables. For the Poiseuille profile, the associated pressure gradient becomes $\mathrm{d} \bar{P} / \mathrm{d} \bar{X}=-8 g_{o} / \pi$, while the pressure gradient with slip flow vanishes as $\bar{X} \rightarrow \infty$. To write the remaining boundary conditions one needs to study the solution to $(6.1)-(6.3)$ as $\bar{X} \rightarrow-\infty$, which matches with the asymptotic results in $\mathrm{M}$ as $x \rightarrow x_{s}$.

\subsection{Solution for $\bar{X} \rightarrow-\infty$}

For $\bar{X} \rightarrow-\infty$ the flow approaches a self-similar solution of the equations, of the type found downstream from region $\mathrm{O}$. In particular, the radial velocity becomes independent of $\bar{X}$, and the axial velocity and the axial pressure gradient increase linearly with distance. Appropriate similarity expansions for the different flow variables are

$$
\left.\begin{array}{l}
U_{t}=-\bar{U}\left(\bar{X}+B g_{o} \ln |\bar{X}|\right)+B g_{o} \tilde{U}+\cdots, \\
V_{t}=\bar{V}\left(1+B g_{o} / \bar{X}\right)+\cdots, \\
\frac{\mathrm{d} \bar{P}}{\mathrm{~d} \bar{X}}=\bar{\Lambda}\left(\bar{X}+B g_{o} \ln |\bar{X}|\right)+\cdots .
\end{array}\right\}
$$

Note that the first terms in the expansions above match with the leading-order solution in $\mathrm{M}$, whereas the remaining terms would enter when matching with the first-order correction, of order $\varepsilon$, which includes a logarithmic singularity as explained above.

Introducing these similarity variables into (6.1) and (6.2) yields at leading order

$$
\begin{gathered}
-\bar{U}+(1 / r)(r \bar{V})_{r}=0, \\
\bar{U}_{r r}+((1 / r)-\bar{V}) \bar{U}_{r}+\bar{U}^{2}=-\bar{\Lambda},
\end{gathered}
$$

to be integrated with boundary conditions given in (6.4) and (6.5):

$$
\bar{U}_{r}(0)=\bar{V}(0)=\bar{U}(1)=\bar{V}(1)=0
$$


for non-slip flow and

$$
\bar{U}_{r}(0)=\bar{V}(0)=\bar{U}_{r}(1)=\bar{V}(1)=0
$$

for slip flow. Use of the similarity stream function $\bar{X} \bar{F}(r)$, such that $\bar{U}=-\bar{F}_{r} / r$ and $\bar{V}=-\bar{F} / r$, reduces the problem to that of integrating

$$
\bar{F}_{r r r}+\bar{F}_{r r}(\bar{F}-1) / r+\bar{F}_{r}\left(1-r \bar{F}_{r}-\bar{F}\right) / r^{2}=\bar{\Lambda} r,
$$

with boundary conditions

$$
\left(\bar{F}_{r} / r\right)_{r}=\bar{F}=0
$$

at $r=0$ and

$$
\left.\begin{array}{ll}
\bar{F}_{r}=\bar{F}=0 & \text { (non-slip flow) } \\
\left(\bar{F}_{r} / r\right)_{r}=\bar{F}=0 & \text { (slip flow) }
\end{array}\right\}
$$

at $r=1$. The equation for $\bar{F}$ is identical to that previously written in (4.11) for the stream function $F$ downstream from region $\mathrm{O}$.

Apart from the solution $\bar{F}=0$ and $\bar{\Lambda}=0$, the problem admits a non-trivial solution for $\bar{A}=-348.59$ (non-slip flow) and $\bar{A}=-162.27$ (slip flow), and the associated velocity profiles $\bar{U}=-\bar{F}_{r} / r$ and $\bar{V}=-\bar{F} / r$ are shown in figure 12 . A shooting method was used, as before, to integrate (4.11). Shooting was initiated at $r \ll 1$, where the solution of the above equation is in general of the form $\bar{F}=\bar{\Lambda} r^{4} / 16+B_{1} r^{2}+B_{2}+B_{3} r^{2} \ln (r)$. The boundary conditions at $r=0$ require that $B_{2}=B_{3}=0$, leaving $B_{1}$ and $\bar{A}$ as shooting parameters to be varied until the two boundary conditions at $r=1$ were satisfied. The values $B_{1}=-17.698$ and $B_{1}=-12.424$ were obtained for non-slip and slip flow, respectively.

As previously mentioned, the first terms in the expansions (6.7) match with the leading-order solution in $\mathrm{M}$, which near the stagnation plane admits the simplified representation $u=\left(x_{s}-x\right) \bar{U}(r), v=\bar{V}(r)$, and $\mathrm{d} p / \mathrm{d} x=\bar{\Lambda}\left(x-x_{s}\right)$. This result can be used to further test the accuracy of the boundary-layer solutions obtained by integrating (5.1) and (5.2). The degree of agreement obtained is illustrated in figures 9 and 12. The linear decrease with distance of the pressure gradient and of the axial velocity seen in figure 9 closely agrees with the asymptotic predictions. Also, the radial velocity distribution near $x=x_{s}$ adjusts to the asymptotic prediction $v=\bar{V}(r)$, a result that is illustrated in figure 12 .

The expansions (6.7) incorporate a higher-order correction $\tilde{U}(r)$ to the axial velocity, which is included to accommodate the non-zero mass flux $g_{o}$, along with appropriate switchback terms (the second terms in the parentheses), which are necessary to provide solvability for $\tilde{U}(r)$. The correction is determined by the linear problem

$$
\begin{aligned}
& \tilde{U}_{r r}+\left(\frac{1}{r}-\bar{V}\right) \tilde{U}_{r}+\bar{U} \tilde{U}=\bar{U}^{2}-\bar{V} \bar{U}_{r},
\end{aligned}
$$

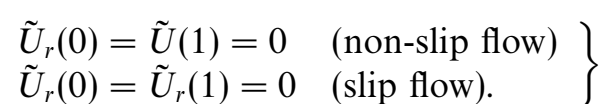

The resulting profile $\tilde{U}(r)$ is shown in figure 13. The constant $B$ appearing in (6.7) is given by $B=\left(\int_{0}^{1} 2 \pi r \tilde{U} \mathrm{~d} r\right)^{-1}$, yielding $B=0.01981$ for non-slip flow and $B=0.01953$ for slip flow.

\subsection{Leading-order solution}

The expressions given in (6.7) for the velocity components complete the boundary conditions needed to integrate (6.1)-(6.3), which are invariant under translations in $\bar{X}$. 

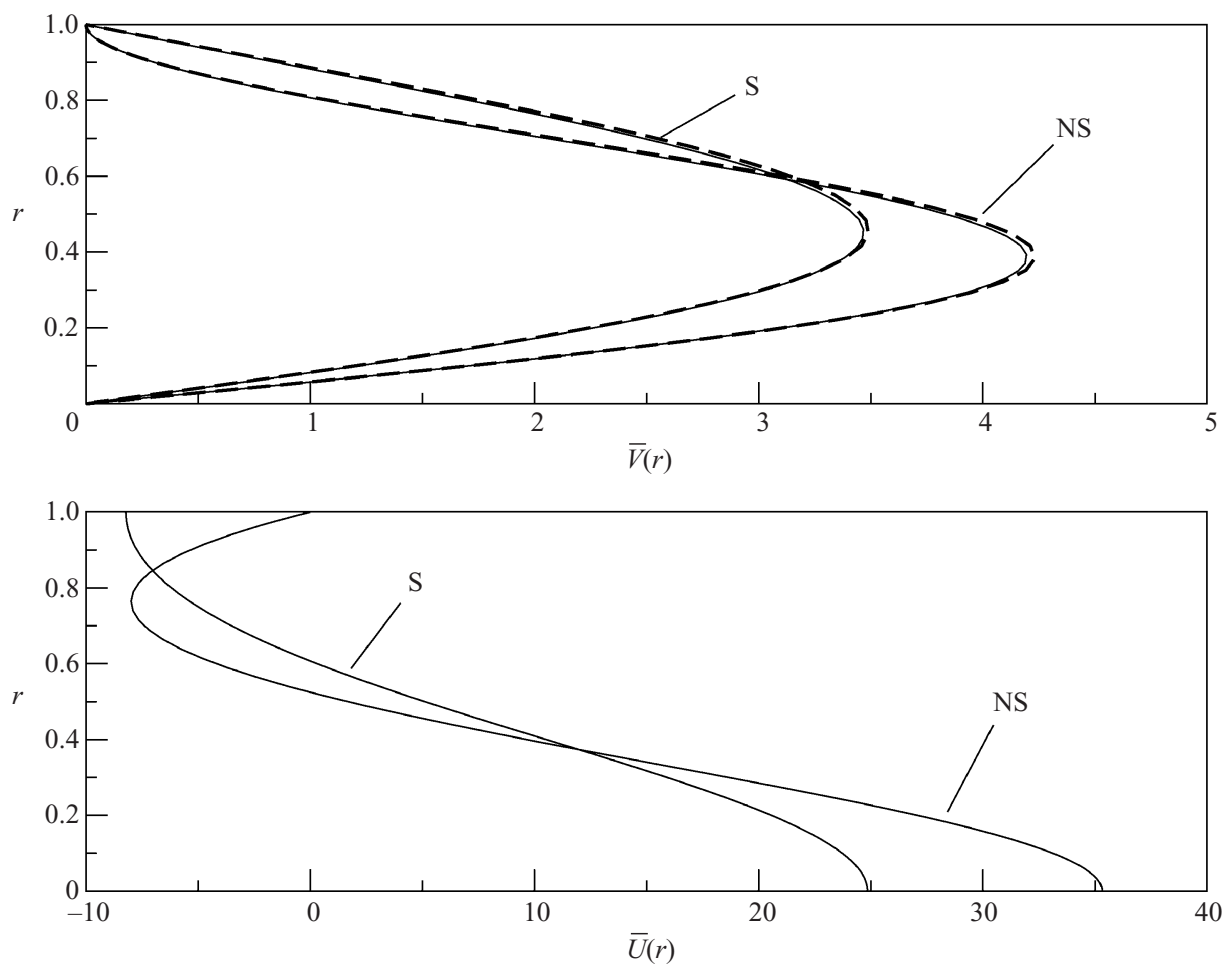

FIGURE 12 . The radial variation of the functions $\bar{U}(r)$ and $\bar{V}(r)$ with non-slip boundary conditions (NS) and with slip boundary conditions (S); the dashed line represents the radial velocity profile of the overall solution for $\varepsilon=0$ evaluated at $x=0.102$ (NS) and at $x=0.129$ (S).

One could arbitrarily choose the origin of $\bar{X}$ incorporating a translation of magnitude $\bar{X}_{T}$ in the boundary condition, by using $U_{t}=-\bar{U}\left(\bar{X}+\bar{X}_{T}+B g_{o} \ln |\bar{X}|\right)+B g_{o} \tilde{U}$ instead of the corresponding expansion shown in (6.7). In the results presented below, this arbitrariness was removed by choosing $\bar{X}_{T}=0$.

The numerical procedure employed is identical to that developed for integrating (4.1)-(4.3). Streamlines of the resulting flow field with non-slip flow at $r=1$ and a Poiseuille inlet velocity profile are exhibited in figure $14(a, b)$ for $R e_{a}=1.0$ and 2.5. The dividing streamline is here assigned the value $\Psi=\varepsilon \psi=0$. Solid lines correspond to positive values of $\Psi$ (recirculating fluid) equally spaced in increments $\delta \Psi=g_{o} /(2 \pi)$, whereas the spacing used for the negative values of $\Psi$ equals $\delta \Psi=g_{o} /(10 \pi)$. As can be seen, a result of our selection for $\bar{X}_{T}$ is that the dividing streamline intersects the wall at an axial location $\bar{X} \neq 0$ which varies with $R e_{a}$. The transition towards the final Poiseuille solution occurs downstream from this location, in a region that becomes shorter as the Reynolds number increases, a behaviour also observed in the numerical computations of the overall problem shown in figure 10.

Results corresponding to $R e_{a} \rightarrow \infty$ are also included in figure 14(c). In that case, (6.1)-(6.3) reduce to the boundary-layer equations, with $\partial \bar{P} / \partial r=0$ replacing (6.3) and with the last term neglected in (6.2). The axial velocity profile given in (6.7) is employed as the initial condition in the integration. As can be seen, the streamlines are similar to those obtained with $R e_{a}=2.5$, indicating that the boundary-layer limit approximates well the structure of the transition region even for values of $R e_{a}$ only 

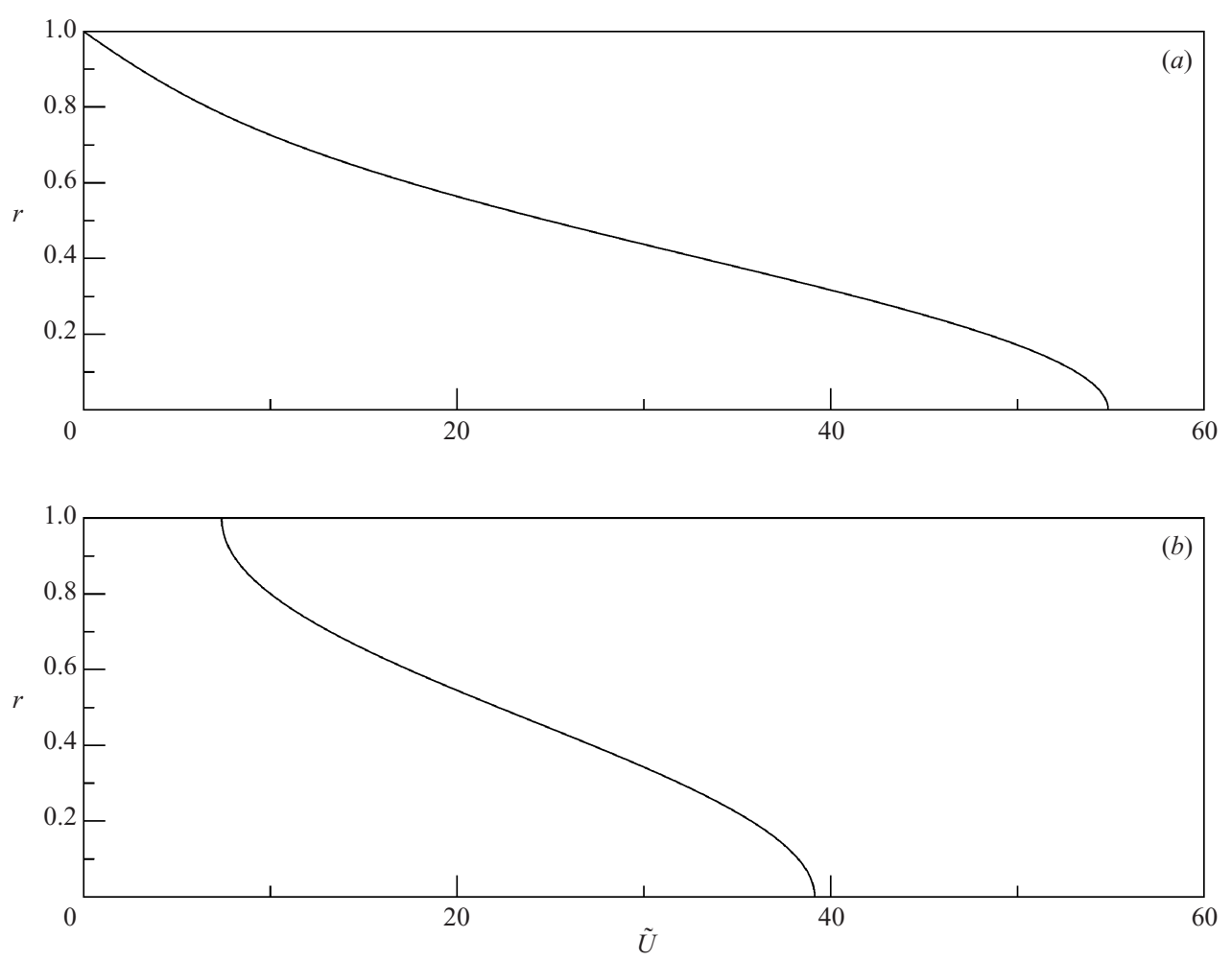

FIGURE 13 . The radial variation of the function $\tilde{U}(r)$ with $(a)$ non-slip boundary conditions and (b) with slip boundary conditions.

moderately large. For completeness, the plot includes axial velocity profiles obtained with the boundary-layer approximation at different downstream locations.

The dependence of the solution on $R e_{a}$ is further illustrated in figure 15, where the axial pressure gradient at the axis (plot $a$ ), at the wall (plot $b$ ), and the velocity distribution along the axis (plot $c$ ) are shown for the three cases of figure 14. The plot reproduces the trends observed previously: the transition to the final Poiseuille solution is more abrupt for larger values of $R e_{a}$, and the quantitative agreement between the boundary-layer results and those of the Navier-Stokes integrations is already apparent for $R e_{a}=2.5$.

Small modifications should be incorporated in the problem formulation to describe the region of streamline deflection in the limit $R e_{a} \ll 1$, a development that parallels the analysis of region $\mathrm{O}$ in this same limit. The characteristic axial length of streamline deflection, $x-x_{e} \sim a$, and the characteristic axial velocity, $u \sim v / a$, must be used in defining appropriate rescaled variables $\tilde{X}=R e_{a} \bar{X}, \tilde{U}_{t}=R e_{a} U_{t}$ and $\tilde{P}=R e_{a}^{2} \bar{P}$. Use of these variables enables the parameter $R e_{a}$ to be scaled out of the Navier-Stokes equations (6.1)-(6.3). Apart from (6.4) and (6.5), the rescaled velocity must satisfy $\tilde{U}_{t}=$ $-\bar{U} \tilde{X}$ and $V_{t}=\bar{V}$ at $\tilde{X}=-\infty$ and $\tilde{U}_{t}=V_{t}=0$ at $\tilde{X}=\infty$, the leading-order boundary conditions obtained by neglecting in (6.6) and (6.7) terms that vanish when $R e_{a} \rightarrow 0$. With a zero mass flux, the leading-order solution must show for $\tilde{X} \rightarrow \infty$ a velocity that decays with $\tilde{X}$ at an exponential rate, eventually giving rise to the Stokes toroidal vortices described by Wakiya (1976). This leading-order description can be expected 

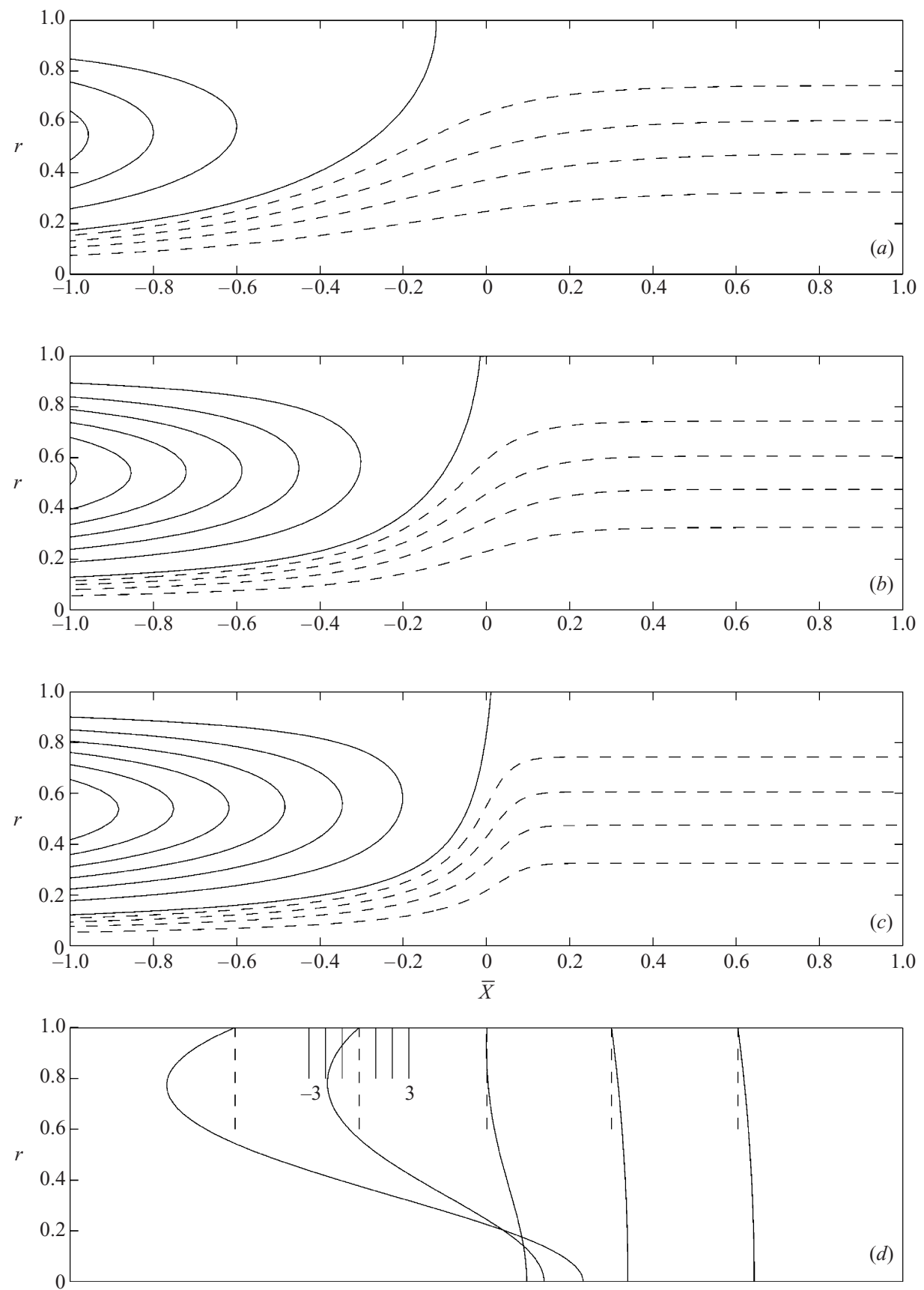

FIGURE 14. Solution in the transition region with non-slip flow at $r=1$ and Poiseuille velocity profile at the inlet for $(a) R e_{a}=1.0,(b) R e_{a}=2.5,(c) R e_{a}=\infty$. Recirculating streamlines (solid lines) correspond to increments $\delta \Psi=g_{o} /(2 \pi)$ from the $\Psi=0$ value of the dividing streamline, while streamlines of escaping fluid (dashed lines) correspond to decrements $\delta \Psi=-g_{o} /(10 \pi)$ from this same value. (d) Velocity profiles for the boundary-layer results are given at $\bar{X}=(-0.6,-0.3,0.0,0.3,0.6)$, with the velocity scale indicated in the plot at $\bar{X}=-0.3$. 

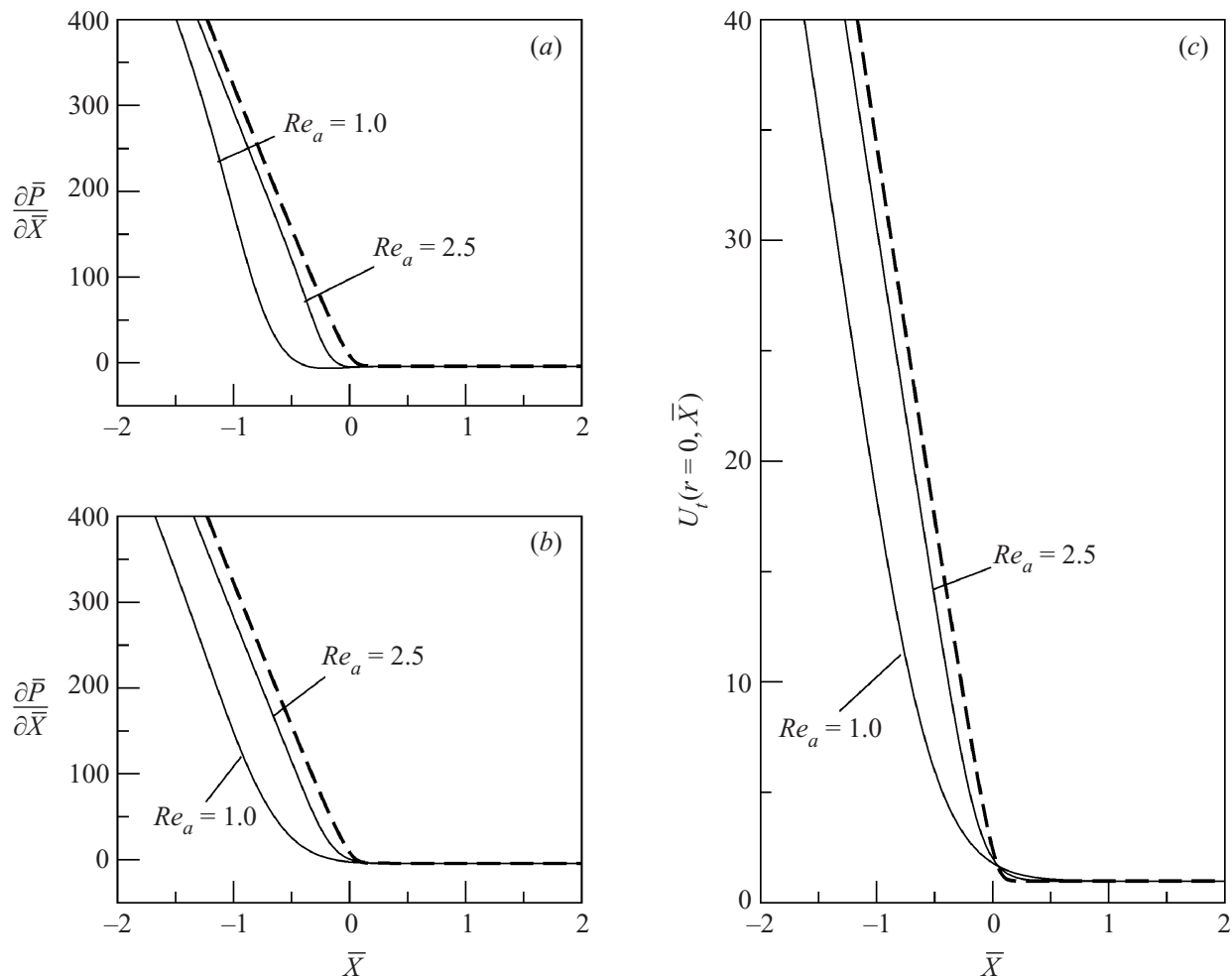

FIGURE 15. The pressure gradients at the wall $(b)$ and at the axis $(a)$, along with the axial velocity distribution along the axis $(c)$ as obtained from the boundary-layer results (dashed lines) and from the full Navier-Stokes problem (solid lines) for the transition region of figure 14.

to fail as $\tilde{U}_{t}$ decays to values of order $R e_{a}$, where the mass flux should be taken into account, eventually leading to either the Poiseuille profile or a uniform velocity profile.

\section{Conclusions}

Round laminar jets with coaxial confinement are investigated when the jet Reynolds number and the expansion ratio are both large quantities. The long recirculating region that forms in this case is seen to be bounded at both ends by relatively short boundary regions. All regions have been described separately for relevant values of the parameters involved, including the distinguished limit $\operatorname{Re}_{j} \varepsilon \sim 1$ for the analysis of the slow fluid motion in the boundary regions. The asymptotic analysis provides in particular quantitative results of practical interest, including the length of the recirculating eddy $(0.106 \sqrt{\mathrm{J} / \rho} a / v$ for non-slip flow and $0.131 \sqrt{\mathrm{J} / \rho} a / v$ for slip flow) and its associated pressure increment $\left(0.593 \mathrm{~J} / a^{2}\right.$ for non-slip flow and $0.318 \mathrm{~J} / a^{2}$ for slip flow). The asymptotic description has been validated through comparisons with results of integrations of the Navier-Stokes equations for moderately large values of $R e_{j}$ and $1 / \varepsilon$.

The analysis presented herein has attempted to address in as much generality as possible the description of confined laminar jets with large expansion ratios. Nevertheless, our work has deliberately ignored some limiting cases that are left for future work, including the analysis of confined and unconfined jets with Reynolds numbers of order unity and smaller. Confined jets with $R e_{j} \gg 1$ and with exponentially large 
expansion ratios such that $\varepsilon \sim \exp \left(-R e_{j}^{2} / 15.28\right)$, when momentum decay in the jet region becomes important, is another limiting case that deserves further attention, since it provides the transition from the confined jets analysed here to the unconfined jets of Schneider (1985).

The type of asymptotic treatment presented here can also be useful to address stability, which should be treated in future work. Also of interest is the extension of the present work to combustion applications, when a compound jet, formed for example by a fuel jet and a coaxial air jet, discharges in the chamber. While a number of papers have been recently published on flame stabilization in axisymmetric free jet flows (see, e.g., Lee \& Chung 1997 and Chen \& Bilger 2000), the effect of confinement on the stabilization mechanism remains unclear. It can be expected that the composition of the recirculating gases plays an important role in the stability of the combustion process, an issue to be clarified in future work.

The extension of the analysis to cover turbulent flows also deserves investigation. The solution of laminar and turbulent free jets is known to be alike, in that, by replacing $v$ by an appropriate eddy viscosity, Schlichting analysis is seen to reproduce well the results of turbulent jets. It can therefore be expected that a similar simple analysis with slip flow considered at the outer wall applies in confined turbulent jets. Matching the recirculating length obtained with the boundary-layer approximation with that found in turbulent flows seems to be an appropriate criterion for the selection of the eddy viscosity in that case.

The authors are indebted to Professor F. Higuera and to Mr J. D. Mellado for enlightening discussions on the subject. The work of A. R. and A. L.S. was supported by the Spanish DGESIC under project number PB98-0142-C04-02, while that of A. L. was supported by the Spanish CICYT under contract no PB94-0400.

\section{Appendix. Boundary-layer numerical scheme}

A pseudo-transient finite-volume scheme was used to integrate the boundary-layer equations in the main region. The conservation equations, written in conservative form, and with a time derivative artificially added to the momentum balance equation, were integrated until convergence to a steady solution was achieved. The time scheme was a generalized implicit Crank-Nicholson scheme in the radial coordinate and explicit in the axial coordinate. Second-order centred schemes were used for the spatial derivatives. This treatment involves the solution of a tridiagonal system of the linear equations for every axial position and time step.

The method employed to solve the problem is equivalent to that of Brady \& Acrivos (1982) . The pressure gradient is obtained as part of the solution, to conserve mass. For the $n+1$ time step $\mathrm{d} p / \mathrm{d} x^{n+1}=\mathrm{d} p / \mathrm{d} x^{n}+\delta(\mathrm{d} p / \mathrm{d} x)$ and $u^{n+1}=u^{\star}+\delta u$, and the time-discretization of the momentum equation is set as

$$
\begin{aligned}
\frac{r\left(u^{\star}-u^{n}\right)}{\Delta t}= & (1-\beta)\left[\frac{\partial}{\partial r}\left(r \frac{\partial u^{\star}}{\partial r}\right)-\frac{\partial\left(r v^{n} u^{\star}\right)}{\partial r}\right] \\
& +\beta\left[\frac{\partial}{\partial r}\left(r \frac{\partial u^{n}}{\partial r}\right)-\frac{\partial\left(r v^{n} u^{n}\right)}{\partial r}\right]-\frac{\partial\left(r u^{n} u^{n}\right)}{\partial x}-r \frac{\mathrm{d} p^{n}}{\mathrm{~d} x}
\end{aligned}
$$

and $\delta u=-\Delta t \times \delta(\mathrm{d} p / \mathrm{d} x)$, where the correction of the axial velocity $\delta u=\varepsilon g_{o} / \pi-$ $\int_{0}^{1} 2 r u^{\star} \mathrm{d} r$ is computed from the mass-conservation condition (2.10). Finally the values of the radial velocity $v^{n+1}$ are calculated with the continuity equation from the values of the axial velocity. 
The value of the $\beta$-parameter used for the Crank-Nicholson scheme was $\beta=0.25$, a compromise between $\beta=0.5$, which tends to produce nonlinear instabilities, and $\beta=0$, corresponding to the full implicit scheme, which yields less accurate results. The axial convective term determines the time step selected according to $\Delta t=C F L \times \Delta x_{\min } /\left|U_{\max }\right|$, with a $C F L=1$ being utilized in the computations. The results presented below correspond to a grid that is uniformly distributed in the axial direction with spacing $\delta x=1.0 \times 10^{-3}$. The radial node distribution is non-uniform, with minimum spacing $\delta r=1.03 \times 10^{-3}$ near the axis and also near the wall. The convergence criterion selected was that the variation of the pressure gradient along the axis at each time step was much smaller than the corresponding integration time step, i.e. $\sum\left|\mathrm{d} p / \mathrm{d} x^{n+1}-\mathrm{d} p / \mathrm{d} x^{n}\right| \ll \Delta t$.

The numerical method was tested extensively. For instance, the accuracy of the final numerical solutions was checked by evaluating the integral form of the momentum equation applied to the recirculating fluid, yielding an error of less than $0.6 \%$ for the calculations shown below. Different modifications were introduced to further validate the numerical scheme proposed. It was seen that streching of the axial coordinate does not provide significant improvement. Alternative discretizations that were tested include a three-level implicit scheme in time for the radial coordinate, and high-order upwind schemes (Drikais \& Tsangaris 1993) for the $x$-convective term. These alternative discretizations enable larger time steps in the computation, but no significant differences were observed in the accuracy of the final results.

To analyse the grid-dependence of the results, the leading-order problem of figure 7 , which was calculated with a grid of $\mathrm{NX} \times \mathrm{NR}=120 \times 75$ nodes, was also computed with a coarser grid of $60 \times 50$ nodes $\left(\delta x=2.0 \times 10^{-3}\right.$ and minimum $\left.\delta r=1.65 \times 10^{-3}\right)$ and with a finer grid of $180 \times 100$ nodes $\left(\delta x=6.67 \times 10^{-4}\right.$ and minimum $\delta r=7.45 \times$ $10^{-4}$ ), yielding an error in the momentum integral balance equal to $1 \%$ and $0.4 \%$, respectively. The values of the maximum pressure gradient $(=10.419,10.492,10.515)$ and eddy length $(=0.108,0.1062,0.1056)$ were seen to vary only by a small amount with grid refinement for the three grids employed, with differences between the two finest grids being smaller than $0.6 \%$. To study the influence of the time step on the final results and the stability of the numerical method, different values of the $C F L$ ranging from 0.5 to 3 were used. Negligible differences in the final results were found for the same grid.

\section{REFERENCES}

Acrivos A. \& Schrader, M. L. 1982 Steady flow in a sudden expansion at high Reynolds numbers. Phys. Fluids 25, 923-930.

Allerborn, N., Nandakumar, K., Raszillier, H. \& Durst, F. 1997 Further contributions on the two-dimensional flow in a sudden expansion. J. Fluid Mech. 330, 169-188.

Batchelor, G. K. \& Gill, A. E. 1962 Analysis of the stability of axisymmetric jets. J. Fluid Mech. 14, 529-551.

Battaglia, F., Kulkarni, A. K., Feng, J., \& Merkle, C. L. 1998 Simulations of planar flapping jets in confined channels. AIAA J. 36, 1425-1431.

Battaglia, F., Tavener, S. J., Kulkarni, A. K. \& Merkle, C. L. 1997 Bifurcation of low Reynolds number flows in symmetric channels. AIAA J. 35, 99-105.

Brady, J. F. \& ACRIVOS, A. 1982 Closed-cavity laminar flows at moderate Reynolds numbers. J. Fluid Mech. 115, 427-442.

Chapman, D. R. 1949 Laminar mixing of a compressible fluid. NACA-TN 1800.

Chen, Y. C. \& Bilger, R. W. 2000 Stabilization mechanisms of lifted laminar flames in axisymmetric jet flows. Combust. Flame 123, 23-45. 
Cherdron, W., Durst, F. \& Whitelaw, J. H. 1978 Asymmetric flows and instabilities in symmetric ducts with sudden expansions. J. Fluid Mech. 84, 13-31.

DrIKaKIS, D. 1997 Bifurcation phenomena in incompressible sudden expansion flows. Phys. Fluids 9, 76-87.

DrIKAKIS, D. \& TSANGARIS, S. 1993 On the solution of the compressible Navier-Stokes equations using improved flux vector splitting. Appl. Math. Modelling 17, 282-297.

Durst, F., Melling, A. \& Whitelaw, J. H. 1974 Low Reynolds number flow over a plane symmetrical sudden expansion. J. Fluid Mech. 64, 111-128.

Durst, F., Pereira, J. C. F. \& Tropea C. 1993 The plane symmetric sudden-expansion flow at low Reynolds numbers. J. Fluid Mech. 248, 567-581.

Fearn, R. M., Mullin, T. \& Cliffe, K. A. 1990 Nonlinear flow phenomenon in a symmetric sudden expansion. J. Fluid Mech. 211, 595-608.

Goldstein, S. 1930 Concerning some solutions of the boundary-layer equations in hydrodynamics. Proc. Camb. Phil. Soc. 26, 1-30.

Hawa, T. \& RusaK, Z. 2000 Viscous flow in a slight asymmetric channel with a sudden expansion. Phys. Fluids 12, 2257-2267.

Hawa, T. \& RusaK, Z. 2001 The dynamics of a laminar flow in a symmetric channel with a sudden expansion. J. Fluid Mech. 436, 283-320.

Klemp, J. B. \& Acrivos, A. 1976 A moving-wall boundary layer with reverse flow. J. Fluid Mech. 76, 363-381.

KumAR, A. \& YAJNIK, K. S. 1980 Internal separated flows at large Reynolds numbers. J. Fluid Mech. 97, 27-51.

Lee, B. J. \& Chung, S. H. 1997 Stabilization of lifted tribrachial flames in a laminar nonpremixed jet. Combust. Flame 109, 163-172.

LESSEN, M. 1950 On the stability of the laminar free boundary between parallel streams. $N A C A-R$ 979.

Macagno, E. O. \& Hung, T-K. 1967 Computational and experimental study of a captive annular eddy. J. Fluid Mech. 28, 43-64.

Milos, F. S. \& Acrivos, A. 1986 Steady flow past sudden expansions at large Reynolds numbers. Part I: Boundary layer solutions. Phys. Fluids 29, 1353-1359.

Milos, F. S. \& Acrivos, A. 1987 Steady flow past sudden expansions at large Reynolds numbers. Part II: Navier-Stokes solutions for the cascade expansion. Phys. Fluids 30, 7-18.

MofFATT, H. K. 1964 Viscous and resistive eddies near a sharp corner. J. Fluid Mech. 18, 1-18.

Mollendorf, J. C. \& Gebhart, B. 1973 An experimental and numerical study of the viscous stability of a round laminar vertical jet with and without thermal buoyancy for symmetric and asymmetric disturbances. J. Fluid Mech. 61, 367-399.

Nathan, G. J., Hill, S. J. \& Luxton, R. E. 1998 An axisymmetric fluidic nozzle to generate jet precession. J. Fluid Mech. 370, 347-380.

Patankar, S. V. 1980 Numerical Heat Transfer and Fluid Flow. Hemisphere.

Patankar S. V. \& Spalding, D. B. 1972 A calculation procedure for heat, mass and moment transfer in three-dimensional parabolic flows. Intl J. Heat Mass Transfer 15, 1787-1806.

Revuelta, A., Sánchez, A. L. \& Liñán, A. 2002 Laminar mixing in diluted and undiluted fuel jets upstream from lifted flames. Combust. Flame, In Press.

Reynolds, A. J. 1962 Observations of a liquid-into-liquid jet. J. Fluid Mech. 14, 552-556.

RusAK, Z. \& HAWA, T. 1999 A weakly nonlinear analysis of the dynamics of a viscous flow in a symmetric channel with a sudden expansion. Phys. Fluids 11, 3629-3636.

SChlichting, H. 1933 Laminare strahlausbreitung. Z. Angew. Math. Mech. 13, 260-263.

SCHNEIDER, W. 1981 Flow induced by jets and plumes. J. Fluid Mech. 108, 55-65.

SCHNEIDER, W. 1985 Decay of momentum flux in submerged jets. J. Fluid Mech. 154, 91-110.

Shapira, M., Degani, D. \& Weihs, D. 1990 Stability and existence of multiple solutions for viscous flow in suddenly enlarged channel. Comput. Fluids 18, 239-258.

WAKIYA, S. 1976 Axysimmetric flow of a viscous fluid near the vertex of a body. J. Fluid Mech. 78, 737-747.

ZAUNER, E. 1985 Visualization of the viscous flow induced by a round jet. J. Fluid Mech. 154, 111-119. 